\title{
NR2A- and NR2B-NMDA receptors and drebrin within postsynaptic spines of the hippocampus correlate with hunger- evoked exercise
}

\author{
Yi-Wen Chen ${ }^{1}$, Hannah Actor-Engel ${ }^{1}$, Ang Doma Sherpa ${ }^{1}$, Lauren Klingensmith ${ }^{1}$, Tara G. \\ Chowdhury ${ }^{1,2}$, and Chiye Aoki ${ }^{1}$ \\ ${ }^{1}$ Center for Neural Science, New York University, 4 Washington PlaceRoom 809, New York, NY \\ 10003, USA \\ ${ }^{2}$ Department of Neuroscience, University of Pittsburgh, Pittsburgh, USA
}

\section{Abstract}

Hunger evokes foraging. This innate response can be quantified as voluntary wheel running following food restriction (FR). Paradoxically, imposing severe FR evokes voluntary FR, as some animals choose to run rather than eat, even during limited periods of food availability. This phenomenon, called activity-based anorexia (ABA), has been used to identify brain changes associated with FR and excessive exercise (EX), two core symptoms of anorexia nervosa (AN), and to explore neurobiological bases of AN vulnerability. Previously, we showed a strong positive correlation between suppression of FR-evoked hyperactivity, i.e., ABA resilience, and levels of extra-synaptic GABA receptors in stratum radiatum (SR) of hippocampal CA1. Here, we tested for the converse: whether animals with enhanced expression of NMDA receptors (NMDARs) exhibit greater levels of FR-evoked hyperactivity, i.e., ABA vulnerability. Four groups of animals were assessed for NMDAR levels at CA1 spines: (1) ABA, in which 4 days of FR was combined with wheel access to allow voluntary EX; (2) FR only; (3) EX only; and (4) control (CON) that experienced neither EX nor FR. Electron microscopy revealed that synaptic NR2A-NMDARs and NR2B-NMDARs levels are significantly elevated, relative to CONs'. Individuals' ABA severity, based on weight loss, correlated with synaptic NR2B-NMDAR levels. ABA resilience, quantified as suppression of hyperactivity, correlated strongly with reserve pools of NR2A-NMDARs in spine cytoplasm. NR2A- and NR2B-NMDAR measurements correlated with spinous prevalence of an F-actin binding protein, drebrin, suggesting that drebrin enables insertion of NR2B-NMDAR to and retention of NR2A-NMDARs away from synaptic membranes, together influencing ABA vulnerability.

\section{Keywords}

Anorexia nervosa; Food restriction; Exercise; Foraging; Receptor trafficking; Eating disorder

Correspondence to: Chiye Aoki.

Y.-W. Chen and H. Actor-Engel contributed equally to the manuscript.

Electronic supplementary material: The online version of this article (doi:10.1007/s00429-016-1341-7) contains supplementary material, which is available to authorized users.

Compliance with ethical standards: Conflict of interest We declare no conflict of interest in relation with the work described. 


\section{Introduction}

Although much of neocortical development reaches completion during the juvenile stage (Hensch and Fagiolini 2005; Aoki and Erisir 2014), development of the hippocampus continues into late adolescence. In the dorsal hippocampus of rats, for example, clearly, definable place cells only emerge towards the end of adolescence, around postnatal day (P) 50 (Martin and Berthoz 2002). Unsurprisingly, for a brain region still developing, the experience of food restriction and exercise during adolescence strongly alters the complexity of dendritic arbors of CA1 pyramidal neurons (Chowdhury et al. 2014a, c; Aoki and Erisir 2014). In this study, we examined whether this experience also influences the expression and localization of NMDA receptors (NMDARs) at axo-spinous synaptic junctions of CA1 pyramidal neurons.

We chose food restriction and wheel access as environmental factors to study experiencedependent plasticity because of our desire to understand the neurobiological basis of anorexia nervosa (AN) using an animal model. When these two environmental factors are applied simultaneously, a rat's voluntary wheel running increases dramatically (Aoki et al. 2016). This food-restriction-evoked hyperactivity, termed activity-based anorexia (ABA), has been used as an animal model for AN (Guisinger 2003; Casper et al. 2008; Gutierrez 2013). AN is the mental illness with the highest mortality rate, estimated to be 200 times higher than the rate of suicide in the general US population (Arcelus et al. 2011; Sullivan 1995). Twin studies indicate that genetic heritability accounts for 50-70\% of this condition, with the rate of occurrence being ninefold higher among women than men (Bulik et al. 2010; Klump et al. 2001; Wade et al. 2000). However, the recent genome-wide association studies (GWASs) of AN have not yet identified genes of interest (Boraska et al. 2014). Despite high rates of relapse ( 25\%) (Hudson et al. 2007; Steinhausen 2002), AN is without accepted pharmacological treatments. This reflects paucity of knowledge regarding its etiology.

ABA is induced readily in wild-type rodents by imposing food restriction in combination with access to a running wheel (Aoki et al. 2014; Wable et al. 2015b). This is an important behavior shared among individuals with AN (Davis et al. 1997), with some studies indicating that all patients exhibit hyperactivity/over-exercise during the course of the illness and/or prior to diagnosis (Kron et al. 1978). Besides hyperactivity, a number of behavioral phenotypes emerge that also resemble the core symptoms of AN: (1) voluntary food restriction, since food-restricted animals paradoxically choose to run on the wheel instead of eating, even during limited periods of food availability (Chen et al. 2016); (2) elevated anxiety, which correlate with rodents' extent of wheel running (Wable et al. 2015b; Kaye et al. 2004); (3) weight loss (Aoki et al. 2012, 2014); and (4) elevated mortality, which is prevented by removing animals from the ABA-inducing environment before weight loss exceeds $25 \%$. These behaviors become evident within $24 \mathrm{~h}$ of food restriction.

While capturing the above symptoms of $\mathrm{AN}, \mathrm{ABA}$ also reveals individual differences in vulnerability, quantified as the extent of food-restriction-evoked hyperactivity and weight loss (Aoki et al. 2014; Chen et al. 2016; Chowdhury et al. 2013; Wable et al. 2015a, b; 
Nedelescu et al. 2016). Since excitability of the hippocampus is strongly linked to stressinduced anxiety (Shen et al. 2007), and the extent of food-restriction-evoked anxiety is correlated with wheel running (Wable et al. 2015b), we hypothesized that individual differences in wheel running might be influenced by the excitability of hippocampal neurons. Therefore, in this study, we determined whether ABA induction evokes changes in the level of NMDARs at axo-spinous excitatory synaptic junctions of CA1 pyramidal neurons in ways that correlate with ABA vulnerability. We used electron microscopic immunocytochemistry (EM-ICC) (Aoki et al. 2009a, b) to characterize the level and location of two NMDAR subunits, the NR2A and the NR2B, within dendritic spines of the CA1 pyramidal neurons in stratum radiatum, where dendritic remodeling during adolescence is robust and responsive to ABA induction (Chowdhury et al. 2014a, c). Given the known distinctions in function that relate directly to the location of NMDARs (Barria and Malinow 2002; Greer and Greenberg 2008; Hardingham and Bading 2010; Petralia et al. 2010; Thomas et al. 2006; Tovar and Westbrook 1999), we chose EM over Western blotting to distinguish the location of immunoreactivity at postsynaptic membranes versus nonsynaptic and extra-sy-naptic portions on spines, and also to differentiate NMDAR levels at excitatory E-to-E synapses (which form on spines of pyramidal cells) from the E-to-I synapses (which form on dendritic shafts of inhibitory interneurons).

This study also aimed to understand the molecular mechanism underlying experiencedependent changes in NMDAR levels at the hippocampus. Drebrin is an F-actin binding protein that is enriched in hippocampal spines and found selectively at excitatory synapses (Aoki et al. 2005). Drebrin is reduced within the brains of patients diagnosed with mild cognitive impairment and Alzheimer's disease [reviewed in (Aoki et al. 2007)]; it is also required for the activity-dependent trafficking of NR2A-NMDARs into and out of dendritic spines (Aoki et al. 2009a). We examined whether drebrin levels correlated with ABAinduced changes in synaptic NMDAR levels. Indeed, our data indicate that they do. To our knowledge, this is the first study indicating that drebrin is involved in the trafficking of both the NR2A- and the NR2B-NMDARs but in opposite directions, together modulating hungerevoked hyperactivity.

\section{Materials and methods}

\section{Animals}

All procedures involving live rats were in accordance with the Institutional Animal Care and Use Committee of New York University (NYU) (Animal Welfare Assurance No. A3117$01)$.

Thirty-two Sprague-Dawley female rats were used for the study and are the same as those used for analysis of noradrenergic axon terminals in the cerebellum (Nedelescu et al. 2016) and of $a 4 \beta \delta-G A B A(A)$ receptors in the hippocampus (Aoki et al., ms submitted). The rearing conditions of these rats were identical to those described previously (Aoki et al. 2012; Chowdhury et al. 2014a, c), except that they were housed at NYU, instead of at the NY Psychiatric Institute. These rats were delivered to NYU as four cohorts of eight animals at postnatal day $(\mathrm{P}) 28$. Upon arrival, the animals were singly housed with ad libitum access to food and water. Starting on P35 or P36 (Experimental Day 1, ED1), all animals were 
handled daily just before the onset of the dark period for body weight and food weight measurements and assigned to one of the following four groups: control (CON), activitybased anorexia (ABA), food-restricted (FR), or exercised (EX) ( $N=8$ per group). Each group comprised two cohorts of four animals that were delivered together to NYU.

From ED1 to the end of ED8, ABA and EX animals were housed in a new cage attached to a running wheel (Model ENV-046, Med Associates, Inc., St. Albans, VT, USA) (Fig. 1a). Wheel counts were measured continuously for eight days (i.e., up to the end of ED8), using the RotoRat software of Med Associates, and manually once per day. One wheel count equaled $0.64 \mathrm{~m}$. CON and FR animals were given a fresh cage at the beginning of ED1, but were not given access to a running wheel. On ED5, food access for the ABA and the FR groups became restricted to $1 \mathrm{~h}$ per day but unlimited in amount, at the beginning of the dark cycle. CON and EX animals were never restricted of food access in amount or with respect to the time of day. All animals were euthanized on ED8 by transcardial perfusion with fixatives while anesthetized with urethane, as described below under the header, "Brain Tissue Preparation" (Fig. 1a).

\section{Information of antibodies used for immunocytochemistry}

The primary antibodies directed against the NR2A and the NR2B subunits of NMDARs were the same ones described previously (Aoki et al. 2009a, b). The antibody directed against the NR2A subunit of the NMDA receptor was produced in rabbit and corresponds to the amino acids $1265-1464$ of the C-terminus of the rodent subunit. The antibody was purchased from Millipore (Billerica, MA, Cat No. 07-632) and corresponds to the stock originally supplied by Upstate Biotechnology (Lake Placid, NY, Cat. No. 07-632). The specificity of this antibody has been demonstrated previously by Western blotting, which shows that this antibody recognizes a single band corresponding to the molecular weight $\sim 170 \mathrm{kD}$ of the NR2A subunit and neither the NR2B nor the NR1 subunits of NMDAR (Rinaldi et al. 2007). A previous study verified that the anti-NR2A antibody recognizes HEK293 cells transfected with NMDAR-NR2A cDNAs and not NMDAR-NR2B cDNAs and that blocking experiments with immunogens generate loss of immunoreactivity (Adams et al. 2004).

We also used a rabbit antibody to detect the NR2B subunit of the NMDAR. This antibody identifies the NR2B subunit C-terminal amino acids 1437-1456

(KFNGSSNGHVYEKLSSIESDV). The anti-NMDAR2B antibody was also purchased from Millipore (Billerica, MA, Cat No. 06-600) and corresponds to the stock originally supplied by Upstate Biotechnology (Cat. No. 06-600). Specificity of this antibody has been demonstrated by Western blotting, which shows that the antibody recognizes a single band from rat brain microsomal preparation at $\sim 180 \mathrm{kD}$, corresponding to the molecular weight of NR2B. Moreover, this antibody does not recognize NR1 or NR2A subunits of the NMDAR (Rinaldi et al. 2007). We previously conducted EM-ICC and determined that these antibodies recognize asymmetric synapses but not symmetric synapses (Aoki et al. 2009b; Fujisawa and Aoki 2003). This antibody has been used to characterize postnatal changes in the level of NR2B-containing NMDARs at asymmetric synapses of the visual cortex. The developmental profile is as described earlier by Western blots (Sheng et al. Nature 368, 144- 
147), namely, there is a greater prevalence within younger forebrain tissue than in mature tissue, thereby differing significantly from the developmental profile of NR2A immunoreactivity (Corson et al. 2009). Additional electron microscopic immunocytochemical studies have revealed that the NR2A and the NR2B antibodies yield distinct subcellular distributions within single brain regions (Fujisawa and Aoki 2003; Aoki et al. 2003,2009 b), indicating that the two antibodies do not cross-react very strongly.

The secondary antibody used for the detection of NMDAR subunits was donkey anti-rabbit IgG conjugated to $10 \mathrm{~nm}$ colloidal gold particles. This was purchased from Electron Microscopic Sciences (Hatfield, PA, Cat. No. 25705).

We have previously described the specificity of the anti-drebrin antibody, M2F6 (Medical and Biological Laboratories, Japan) (Aoki et al. 2005). This monoclonal antibody recognizes both the $\mathrm{E}$ and $\mathrm{A}$ isoforms of drebrin. Upon genetic deletion of drebrin A, M2F6 recognizes a single band by Western blot of mouse brain homogenates, corresponding to drebrin $\mathrm{E}$ (Aoki et al. 2009a). This antibody was produced using the protein, drebrin, purified electrophoretically from the soluble fraction of 11 day-old chick embryo brains as immunogen.

\section{Brain tissue preparation}

All rats were sacrificed with transcardial perfusions and were prepared as described previously (Aoki et al. 2012, 2014). Perfusions were performed between $4 \mathrm{~h}$ and $30 \mathrm{~min}$ prior to the beginning of the animals' dark cycle. Animals were first anesthetized by intraperitoneal injection with urethane $(0.75 \mathrm{cc} / 100 \mathrm{~g}$ body weight, $34 \% \mathrm{w}: \mathrm{v})$. They were perfused with transcardial fixatives consisting of $4 \%$ paraformaldehyde buffered with $0.1 \mathrm{M}$ phosphate buffer (PB) (pH 7.4). Glutaraldehyde was not used until post-embed processing to preserve the antigenicity of the brain tissue.

Brains were post-fixed in the same fixative after extraction for $9 \mathrm{~h}$. They were then coronally sectioned on a vibratome at a thickness of $40-60 \mu \mathrm{m}$. All tissue sections were then stored at $4{ }^{\circ} \mathrm{C}$ in saline $(0.9 \% \mathrm{NaCl})$ buffered with $0.01 \mathrm{M} \mathrm{PB}$ and preserved with $0.05 \%$ sodium azide(PBS-azide) to prevent bacterial growth. Sections containing the dorsal hippocampus were separated into separate wells for further processing in preparation for post-embed gold immunolabeling.

\section{Immunocytochemistry}

Glutaraldehyde, osmium tetroxide, paraformaldehyde, and EMBED-812 were purchased from EMS. Bovine serum albumin (BSA) was purchased from Sigma Chemicals (St. Louis, MO). Previously described procedures were followed for detecting NR2A and NR2B subunits of NMDARs by the post-embed immunogold (PEG) procedure (Aoki et al. 2000, 2003, 2009a, b; Fujisawa and Aoki 2003), including the use of the same antibodies (Cat. Nos. 07-632 and 06-600 from Millipore, Billerica, MA for detecting NR2A and NR2B subunits, respectively).

Two vibratome sections per animal, randomly picked from multiple vibratome sections, were post-fixed with $2 \%$ glutaraldehyde in PBS for $10 \mathrm{~min}$, then processed by the osmium- 
free procedure for electron microscopy (Phend et al. 1995), strictly in parallel, through the steps for embedding vibratome sections in plastic.

We ran three PEG immunocytochemistry sessions on separate days. This is because pilot PEG immunocyto-chemistry sessions indicated that ultrathin sections collected on grids must be subject to primary antibody incubations starting the fifth to eighth hour after collection. Grids incubated in antibody solutions less than $5 \mathrm{~h}$ after ultrathin-section collections resulted in ultrathin-section floating off from the formvar-coated grids. Grids that air-dried for longer than $8 \mathrm{~h}$ (e.g., overnight) resulted in noticeably reduced immunoreactivity to the antibody. This led to the constraint of preparing all grids within a 3$\mathrm{h}$ period. The highest number of plastic-embedded blocks that could be ultrathin-sectioned during a 3-h period was 13. The "ABA experiment" session ran grids from all eight ABA brain tissue in parallel with grids prepared from four CON brain tissues. The "EX experiment" session ran grids of all eight EX brain tissue in parallel with grids of five CON brain tissues. The "FR experiment" session ran grids of all eight FR animals in parallel with grids of five CON animals (Table 1). From one plastic-embedded block containing one vibratome section, five EM grids were prepared such that two could be allocated for NR2A immunolabeling, two for NR2B immunolabeling, and the fifth grid for the no-antibody control. The fifth grid underwent all identical procedures used for the NR2A and NR2B immunolabeling procedures, except that no primary antibody was included. The post-embed immunogold labeling procedure was exactly as described previously (Aoki et al. 2009b). Antibody concentrations were $10 \mu \mathrm{g} / \mathrm{ml}$ for both the NR2A and NR2B antibodies, applied overnight, while the secondary antibodies were applied at a concentration of 1:100 for $1 \mathrm{~h}$ at room temperature.

In most cases, only a single grid, randomly picked from a pair of grids immunolabeled for the NR2A or the NR2B subunit and typically containing a single large ultrathin section that spanned the dorsal CA1-CA3 and upper blade of the dentate gyrus, from each animal was analyzed. CA3 and dentate gyrus were included in the ultrathin section to provide anatomical landmarks for identifying CA1. For quantitative analysis, the only anatomical region analyzed was stratum radiatum of CA1. A second NR2A- or NR2B-immunolabeled grid was analyzed in cases, where the first randomly picked grid had a tear over the region spanning stratum radiatum of the CA1, making quantitative analysis impossible. Therefore, for most animals, only a single block containing a single vibratome section was used to obtain electron microscopic images.

Drebrin within dendritic spines was detected by the HRP-DAB procedure, prior to embedding in plastic, as described previously (Aoki et al. 2005), using the antibody M2F6 (Medical and Biological Laboratories, Japan, Lot 041) at a dilution of 1:120. Tissue of all eight ABA animals and four out of the eight CON animals (randomly picked) were processed concurrently. Lead citrate counterstaining was omitted to optimize the detection of HRP-DAB reaction product reflecting drebrin immunoreactivity over ultrathin sections with minimal contrast. 


\section{Electron microscopy: digital image acquisition and region selection}

Digital images from stratum radiatum of the dorsal CA1 were taken with a Hamamatsu CCD camera, attached to a JEOL 1200XL electron microscope, using a software developed by AMT (Boston, MA), or were taken using AMT's XR80 camera system. To ensure the least amount of bias, all microscopists were kept blind to the group of the animal (ABA, CON, FR, and EX) during image acquisition and quantitative analysis of the EM images. Images were acquired and analyzed, strictly in the order of encounter, so as to minimize any selection bias for synapses with high or low levels of immunoreactivity. Spines were identified based on their thick postsynaptic density (PSD), the absence of mitochondria, and the absence of microtubules. Synaptic cleft positions were identified based on the presence of thick PSDs. Parallel alignment of the postsynaptic membrane with the vesicle-containing presynaptic terminal's plasma membrane served to confirm the position of the synaptic cleft, but it was not required for identifying asymmetric excitatory axo-spinous synapses (Peters et al. 1991). Images were analyzed and annotated using Adobe Photoshop (version CS2).

\section{PEG particle analysis-general information}

EM grids that were processed for PEG immunolabeling procedure in the absence of the primary antibody yielded $<5 \%$ labeling over axo-spinous synapses, regardless of the animal group to which the tissue belonged. This level was considered background. The occurrence of $\geq 1$ PEG particle was considered immuno-positive for spine labeling. We quantified levels of PEG particles that reflected NR2A and NR2B immunoreactivity at each of the eight mutually exclusive locations at or near axo-spinous synapses (Fig. 2). We also used the values of categories that were condensed (Fig. 2; Tables 2, 3, 4). "Anywhere pre/post" was as an all-inclusive category, reflecting quantitative measurements revealed routinely by immunofluorescence. PEG particle analyses were conducted, using either animals or synapses as independent units.

\section{PEG particle analysis considering animals as independent units for comparisons to individual animal's body weight change and/or wheel running}

-For each group of ten synapses encountered, the proportion of synapses labeled at each of the eight axo-spinous locations was assessed. The per-10-synapses level (number) of PEG particles located at each of the eight axo-spinous locations was also assessed. For each tissue (i.e., animal), this assessment of the level and proportion of synapses labeled at particular axo-spi-nous sites was repeated approximately 17-20 times, by examining approximately 170-200 synapses per animal, to obtain an averaged value for that animal. All the averaged values belonging to a particular PEG immunolabeling session (for example, the averaged values of eight $\mathrm{ABA}$ and four $\mathrm{CON}$ animals that were run in parallel in the "ABA experiment" session) were normalized to a single value corresponding to the average of the values obtained from the $\mathrm{CON}$ tissue of that particular session. Note that the averaged value from each CON animal was also converted to a normalized value, using the single normalization value corresponding to the average of the values obtained from the CON animals specific for that session. We investigated the correlation between normalized $\mathrm{NR} 2 \mathrm{~A} / \mathrm{B}$ immunoreactivities to individual animal's extent of wheel running (EX and ABA groups) or weight changes induced by food restriction (FR and ABA groups). 


\section{PEG particle analysis considering synapses as an independent units, to assess the main effect of food restriction or exercise by two-way ANOVA-To}

determine the environmental effects on NR2A- and NR2B-containing NMDAR localization to excitatory synapses of the hippocampus, we normalized the per-10-synapses PEG particle levels and proportion of spines labeled at each of the eight axo-spinous locations, using a normalization scheme that was similar to that described above. Specifically, for the "ABA experiment", the first step was to calculate the average of the values of PEG particle levels or the proportion of synapses labeled at each subcellular domain of the four CON tissue that were run in parallel with the eight ABA tissue. These values for each subcellular domain, averaged across all of the CON tissue for that session, were used to normalize the PEG particle values of the respective subcellular domains of synapses from all of the eight ABA and the four CON tissues that were immunolabeled in parallel. The same steps of normalization were followed to convert all values from the remaining four sets of tissue: FR, $\mathrm{EX}$, and CON tissues run in parallel with FR, and CON tissue run in parallel with EX. In this way, synaptic values obtained from the tissue of ABA, FR, and EX animals became normalized to the $\mathrm{CON}$ values that were specific to each of the three experimental sessions. Since these steps yielded three sets of CON values (those that ran in parallel with ABA, with FR, and with EX), the values for each CON animal, to be used for two-way ANOVA, were calculated to be the average of the normalized values obtained across the different experimental sessions.

These per-10-synapses values from all animals of the same group were pooled, so as to obtain a single mean value that related to the environmental treatment (e.g., $N=137$ for per-10-synapses, representing 1370 synapses analyzed from ABA tissue; and $N=68$ for per-10-sy-napses, representing 680 synapses analyzed from 4 CON tissue, for the NR2A immunocytochemistry, Table 1). Sample sizes from each animal varied by less than $4 \%$ (Table 1). Two-way ANOVA tests were used to compare the normalized level of NR2A or NR2B immunoreactivity at each axo-spinous synaptic location across the four experimentally reared groups, consisting of ABA, FR, EX, or CON, followed by Fisher's Least Square Difference (LSD) post hoc analysis. Two-way ANOVA test was used to determine whether there was a main effect of exercise or food restriction or whether the two main effects interacted.

Because all values in the graphs are shown as normalized values, the average PEG values of each subcellular domain of CON tissue prior to normalization are presented in Table 2, to reveal PEG levels that differ across the subcellular domains.

Quantitative analysis of drebrin immunoreactivity-PSD (postsynaptic density) profiles of spines were categorized into three: intensely immunolabeled, moderately immunolabeled, or unlabeled. To validate this categorization, a subset of the micrographs was also subjected to mean gray-value measurements. Mean gray values were measured using the tools of 'select' and 'measure' of Image J (version $1.50 \mathrm{~g}$ ). The mean gray-value measurements were randomly sampled from 11 micrographs of ABA tissue (from $3 \mathrm{ABA}$ animals) and 10 micrographs of CON tissue (from $3 \mathrm{CON}$ animals). The formula, [(Mean Gray Value of PSD) minus (Mean Gray Value of Mitochondrion in the immediate vicinity)] divided by (Mean Gray Value of Mitochondrion in the immediate vicinity) was used to 
assess the level of immunolabeling above the mitochondrial 'background' electron density, then to normalize for slight variations in the mean gray values across micrographs arising from differences in the thickness of sections or camera settings.

For each of the eight ABA tissues and four randomly picked CON tissues, spines were assessed for drebrin immunoreactivity, strictly in the order encountered along the EMBEDtissue interface until 96 spines were analyzed. For each group of 12 spines analyzed, the proportion of spines with intensely labeled PSDs was assessed. This assessment was repeated eight times per animal, so as to obtain the mean \pm SEM values of the proportion of spines intensely immunolabeled for drebrin. The micrographs were captured at a magnification of 40,0009, spanning approximately $312.66 \mu \mathrm{m}^{2}$ of neuropil area per animal. To determine whether the environmental effect of ABA upon NR2A- and NR2B-NMDAR localization within spines was influenced by the presence of drebrin, Pearson's correlation analyses were run.

\section{Statistics}

For data pertaining to NR2A and NR2B immunoreactivity, two-way analysis of variance (ANOVA) was used to evaluate the significance of the differences among the four groups, using food restriction and exercise as the two factors, followed by Fisher's LSD post hoc analysis. Pearson's correlation analysis was conducted for relating NR2A and NR2B immunoreactivity to body weight and wheel running measurements and for relating drebrin immunoreactivity to NR2A and NR2B immunoreactivity. One-way ANOVA was used to test significance of the difference in mean gray values of normalized intensity between drebrinimmunolabeled PSDs and mitochondria. Unpaired $t$ test was used to evaluate the significance of difference between ABA and CON groups' drebrin immunoreactivity. For all tests, $p$ values $<0.05$ were considered statistically significant. The statistical softwares used were either Statistica (Tulsa, OK, version 12) or Prism (La Jolla, Calif., versions 6.0 and 7.0).

\section{Results}

\section{Weight loss by the ABA and the FR groups is initially similar}

During the first four experimental days, the average body weight of all four groups increased steadily (Fig. 1b). Average body weights of the CON and the EX groups increased linearly for the entire experimental period, with no significant difference resulting from wheel access (Fig. 1b). During the first three food-restricted period (ED5-ED7), ABA group's average weight did not differ significantly from the FR group's, but both differed significantly from the CON and the EX groups' (Fig. 1b). Individuals within the FR and the ABA groups exhibited variable weight loss over the entire food-restricted period (ED5-ED8), ranging from 11 to $23 \%$ for the ABA group (Fig. 1e) and 11 to $18 \%$ for the FR group (Fig. 1d). As a group, the FR animals began to diverge from the ABA animals toward the end of the foodrestriction period, such that the average weight loss during the last day of food restriction was significantly different between the two groups (Fig. 1b; mean \pm SD of $-2.9 \pm 3.20 \mathrm{~g}$ for $\mathrm{ABA}, 1.72 \pm 1.050 \mathrm{~g}$ for $\left.\mathrm{FR}, t_{14}=-3.87, p=0.005\right)$. 


\section{Food restriction increases voluntary wheel running by the $A B A$ animals}

Prior to food restriction, seven out of the eight animals assigned to the ABA group and seven out of the eight animals assigned to the EX group exhibited an increase in voluntary wheel running, reflecting their acclimation to the apparatus and the appetitive nature of wheel running (Meijer and Robbers 2014). Within both groups, there were individuals that barely increased wheel running (Fig. 1f, g). When food restriction began on ED5 for the ABA group, a difference in the extent of wheel running between the two groups emerged (mean \pm $\mathrm{SD}$ of $6.80 \pm 3.60 \mathrm{~km}$ for the ABA group; $3.49 \pm 2.07 \mathrm{~km}$ for the EX group) with this difference reaching statistical significance by ED7 (Fig. 1c).

\section{NR2A and NR2B immunoreactivity rise at the postsynaptic membrane following ABA induction}

PEG particles reflecting NR2A immunoreactivity were detectable over PSDs, as well as in the cytoplasm of spines and over axon terminals forming axo-spinous asymmetric synaptic junctions (Fig. 2; Table 2). ABA tissue exhibited significantly greater levels (Fig. 3a) and proportion (Supplemental Fig $1 \mathrm{~A}$ ) of spines immunolabeled for NR2A at the postsynaptic membrane compared to CON and EX tissue. The level (Fig. 3a) and proportion of spines (Supplemental Fig. 1A) labeled at the postsynaptic membrane were not altered by as much for the FR or the EX groups.

EX spines exhibited an increase in PEG particle labeling and in the proportion of spines labeled at extra-synaptic plasma membranes (Fig 3a; $p<0.05$ ). Two-way ANOVA revealed a significant interaction of the food-restriction and wheel-access effects, since the addition of the food-restriction paradigm to the wheel-accessed animals (ABA) resulted in a reduction of extra-synaptic labeling, opposite to the effect of exercise alone. These findings indicate that while exercise alone directs NR2A-containing NMDARs to the spine plasma membrane, the additional environmental effect of food restriction combined with exercise brings NR2A-containing NMDARs to the postsynaptic membrane, possibly through lateral diffusion of the NR2A-NMDARs at the extra-synaptic plasma membrane. Unexpectedly, cytoplasmic labeling was not decreased, and instead displayed a non-significant increase. This may be because the cytoplasmic pool is three times larger than the extra-synaptic pool, as is evident by comparing the PEG labeling level across those two subcellular domains of CON tissue (Table 2, "PEG particle number per-10 synapses"). Exercise alone also increased presynaptic NR2A immunoreactivity, while ABA did not (Fig. 3a).

NR2B-PEG also occurred throughout the axo-spinous synapse, within both the axon terminals and dendritic spines (Table 2). ABA induction increased NR2B-PEG particle levels at the postsynaptic membrane (Fig. 3b, $p<0.05$ ), the presynaptic membrane (Fig. 3b, $p<0.001$ ), throughout the axon terminal ("anywhere pre", Supplemental Fig. 2, $p<0.001$ ), and throughout the spine ("Anywhere post", Supplemental Fig. 2, $p<0.005$ ). Of these changes, the postsynaptic membrane level was most notably increased by ABA: the increases by food restriction alone (FR) or wheel access alone (EX) were less and did not reach statistical significance (Fig. 3b). Food restriction alone (FR) or with exercise (ABA) evoked an increase of presynaptic NR2B immunoreactivity, in contrast to the postsynaptic 
NR2B that required the combination of food restriction and exercise to induce increases that were significant.

\section{Inter-animal differences in ABA animals' weight loss and wheel running activity are related to NR2A in the spine cytoplasm but not at the postsynaptic membrane}

As noted in Fig. 1, ABA vulnerability, quantified based on the extent of food-restrictionevoked body weight loss (Fig. 1e) or hyperactivity (Fig. 1g), varied across individuals. To determine whether these ante mortem physiological signatures relates to inter-animal differences in NR2A immunoreactivity at or in the vicinity of axo-spinous synaptic junctions, we investigated the correlations of immunolabeling at each of the synaptic compartments with measurements of body weight changes and wheel running during the food-restricted period. Outcome of the entire analysis is shown in Table 3. The description that follows highlights correlations of ABA vulnerability to synaptic NR2A immunoreactivity and additional correlations that were strong, amongst all correlational analyses that were conducted.

Weight changes correlate with cytoplasmic NR2A-Inter-animal differences in food-restriction-evoked weight loss correlated most strongly with the cytoplasmic, non-activatable pool of NR2A within spines of the ABA tissue. The positive correlation indicated that those animals with minimal loss of body weight during the food-restricted period exhibited the highest levels of cytoplasmic NR2A. This correlation was evident, whether measuring absolute (Fig. 4a, $R=0.84 ; p<0.01$ ) or normalized (Table $3, R=0.80 ; p=0.02$ ) weight changes. Postsynaptic NR2A immunoreactivity did not correlate with weight changes (Fig. $4 \mathrm{~b} ; R=-0.39 ; p=0.34$ for correlation to absolute weight change and Table 3 , $R=-0.16 ; p=0.71$ for correlation to normalized weight change). Unlike the outcome observed for the ABA tissue, correlation analysis of tissue from the FR group of animals revealed no relationship between their weight losses and NR2A immunoreactivity in the spine cytoplasm, even though FR animals lost similar amounts of body weight (Fig. 4a, $R=$ $0.34 ; p=0.42$ for absolute weight changes; Table $3, R=0.32, p=0.44$ for normalized weight loss). This lack of correlation of the cytoplasmic NR2A within FR tissue suggested that the localization of NR2A-containing NMDARs to the spine cytoplasm was not driven by the food-restriction-evoked weight loss, but reflects the combined effect of food restriction plus hyperactivity evoked by it.

Wheel running correlates with cytoplasmic NR2A-Cytoplasmic NR2A labeling within dendritic spines was strongly and negatively correlated with ABA individuals' wheel running, whether measuring the average of the four days of the food-restricted period (Fig. $4 c)$ or only of their food-restriction-evoked increase in running, relative to the pre-foodrestriction baseline level of activity (Table 3 ). The negative correlations indicate that ABA animals responding minimally to the food-restriction treatment expressed the highest levels of NR2A immunoreactivity in the spine cytoplasm. In contrast, postsynaptic NR2A immunolabeling did not correlate with any measure of the wheel activity on any days of the ABA animals (Fig. 4d, $R=-0.00 ; p=0.99$; Table 3). 
We next determined the extent to which wheel running, without food restriction, could contribute to the correlation with spine cytoplasmic NR2A immunoreactivity. The increase in running by animals of the EX group during ED5 and ED6 (corresponding to the first $48 \mathrm{~h}$ of food restriction for the ABA group) correlated significantly and negatively with cytoplasmic NR2A immunolabeling within spines (Table $3 ; R=-0.77, p=0.03$ ), as did the cytoplasmic NR2A labeling for the ABA group (Table $3, R=-0.74, p=0.035$,). However, this relationship did not continue as robustly for the last $48 \mathrm{~h}$ of running by the EX group, thereby bringing the correlation down for the average of the last 4 days (Fig. $4 \mathrm{c}, R=-0.57$; $p=0.14$ ). This might indicate that the extent of running contributed to the rise of NR2A immunoreactivity in the spine cytoplasm but had a much greater, sustained effect for the ABA group than for the EX group.

Together, these data indicate that ABA animals with the highest levels of NR2A immunoreactivity in the spine cytoplasm exhibited both signatures of resilience: minimal weight loss and minimal hyperactivity in response to food restriction. Thus, although functionally dormant, the cytoplasmic pool reflects an active process underlying ABA resilience, while the postsynaptic NR2A immunoreactivity does not.

\section{Inter-animal differences in postsynaptic NR2B immunoreactivity relate to weight loss but not to wheel activity}

NR2B immunoreactivity did not correlate to ABA animals' wheel running, whether measuring the total or average running during the entire food-restricted period (Fig. 5c, d). Because the cytoplasmic NR2A immunoreactivity correlated differently for the EX group across the first compared with the last $48 \mathrm{~h}$, we sought to determine whether these measures correlated in any way with NR2B immunoreactivity as well. This did not reveal any correlation (Table 4). On the other hand, NR2B immunolabeling, specifically at the postsynaptic membrane, correlated with individual animals' degree of weight loss. This correlation to the postsynaptic NR2B immunolabeling was the strongest when compared to the degree of weight loss on ED8 (the last day of food restriction) (Fig. 5e, $R=-0.71 ; p=$ $0.05)$ and was moderately correlated also to weight changes over the entire food-restricted period (Fig. 5b, $R=-0.66 ; p=0.08$ ). No correlation was found between weight loss on ED8 and the extra-synaptic portions of the spine plasma membrane (Table $4, R=-0.01 ; p=$ $0.97)$, indicating that the correlation was specific to the postsynaptic plasma membrane. All FR animals gained weight on ED8 and these changes did not correlate with postsynaptic NR2B immunolabeling (Fig. 5e, $R=0.18 ; p=0.72$ ). Unlike the pattern observed for NR2A immunoreactivity, the cytoplasmic NR2B immunoreactivity within spines did not correlate with body weight changes (Fig. 5a) or average wheel running during the food-restricted period (Fig. 5c).

There also was a significant correlation between the level of PEG particles in axon terminals ('anywhere pre', Fig. 2) and weight loss of ABA animals on the last day of food restriction (Table 4, $R=-0.74 ; p=0.035$ ), although this correlation was not driven by levels of NR2B immunoreactivity specifically at the presynaptic membrane (Table $4, R=-0.35, p=0.39$ ) ("Weight change on ED8," Table 4). Such a correlation with axon terminal labeling was not evident for the FR group, whether examining weight change on the last day of food 
restriction (Table 4, $R=0.22 ; p=0.63$ ) or weight on the last day of food restriction (Table 4, $R=-0.51 ; p=0.24)$.

Comparisons of the FR tissue to the ABA tissue revealed two additional differences in correlations with body weights. Although postsynaptic NR2B immunoreactivity did not correlate to weight lost during food restriction, it correlated significantly and negatively to FR groups' body weight on ED8 (Table 4, $R=-0.79 ; p=0.035$ ). No such correlation was found for the ABA group (Table $4, R=-0.09, p=0.83$ ). We wondered whether postsynaptic NR2B immunoreactivity might be related to general body maturation. This was not the case, since EX animals, which gained body weight steadily throughout the experimental period, exhibited no correlation of their body weight to postsynaptic NR2B (Table $4, R=0.29 ; p=$ $0.48)$.

These analyses reveal that $\mathrm{ABA}$ individuals that could minimize weight loss effectively by eating more during the limited hours of food access exhibited the lowest NR2B-levels postsynaptically (Fig. 5e) and anywhere within spines (Table 4). These correlations are not evident within spines of animals that experience exercise only or food restriction only.

\section{Drebrin immunoreactivity at spines and its relation to NR2A- and NR2B-subunit immunoreactivity}

Cytoplasmic NR2A was strongly negatively correlated with postsynaptic NR2B, the two subcellular domains that exhibited correlation to ABA vulnerability measurements (Fig. 5f, $R=-0.80, p<0.05$ ). This correlation suggests that one pool of NMDARs may be influencing the localization of the other and/or that the two pools are co-regulated by a common cytoplasmic protein. One such candidate protein is drebrin, previously shown to be involved in synaptic protein trafficking of NMDARs and F-actin within dendritic spines (Aoki et al. 2005, 2007, 2009a). This idea was tested by examining drebrin immunoreactivity within dendritic spines of $\mathrm{ABA}$ and $\mathrm{CON}$ tissue from stratum radiatum of the dorsal hip-pocampal CA1.

Initially, we noted that drebrin immunoreactivity over PSDs varied in intensity across synapses, even among those that were located within a few micrometers from one another (Fig. 6a, b). Since drebrin is known to be absent from the mitochondrial matrix, we categorized the intensity of drebrin immunoreactivity over PSDs into three groups: intensely immunolabeled, moderately immunolabeled and unlabeled, using mitochondrial profiles in the immediate vicinity as a local standard representative of an unlabeled profile. We confirmed statistically significant difference in gray values between intensely labeled versus unlabeled or moderately labeled PSDs, with averaged values of $0.06 \pm 0.03$ for unlabeled PSDs, $0.27 \pm 0.03$ for moderately labeled PSDs, and $0.54 \pm 0.02$ for intensely labeled PSDs (Fig. 6c. These group differences were highly significant $(p<0.0001)$ from one another. As expected, the difference in mean gray values between the unlabeled PSDs and mitochondria was small (Fig. 6c). The individual gray values of the intensely labeled and unlabeled PSDs did not overlap at all, confirming that the categorization of the intensely labeled PSDs was sufficiently reliable to allow for quantification. Therefore, we proceeded to compare the proportion of intensely labeled PSDs across ABA versus CON groups. These analyses were conducted while keeping the experimenter blind to the groups. 
For both the ABA and CON tissue, greater than $90 \%$ of the asymmetric axo-spinous synapses exhibited drebrin immunoreactivity over PSDs at moderate or intense levels. The group mean values of the proportion of PSDs with intense immunoreactivity for drebrin were significantly greater for the ABAs than the CONs (Fig. $6 \mathrm{~d}, p=0.009$ ). At the same time, the proportion of spines with intense immunoreactivity for drebrin at PSD varied widely across ABA animals-30 to $68 \%$. Inter-animal differences in the frequency of the intensely immunoreactive PSDs correlated negatively and significantly with cytoplasmic NR2A immunoreactivity (Fig. 6e, $R=-0.78 ; p<0.05$ ) and trended toward a positive correlation with postsynaptic NR2B immunoreactivity $(R=0.65 ; p=0.08$, indicated by a dashed line in Fig. 6f). By comparison, the correlation between postsynaptic NR2A immunoreactivity to the frequency of the intensely drebrin-immunoreactive PSDs was much weaker $(R=0.34 ; p=0.4130)$.

Altogether, these correlation analyses revealed that ABA vulnerability, characterized by larger weight loss and more severe hyperactivity, is associated with higher levels of postsynaptic NR2B immunoreactivity, higher proportions of intensely drebrinimmunoreactive PSDs, and lower levels of cytoplasmic NR2A immunoreactivity (Fig. 7).

\section{Discussion}

During adolescence, food restriction and exercise, separately and together, strongly influence dendritic branching of CA1 pyramidal neurons (Chowdhury et al. 2014a). This study reveals that amidst these dendritic branch changes, ABA also evokes redistribution of NMDARS, together with the F-actin binding protein drebrin at axo-spinous junctions in ways that are different for NR2A-containing and NR2B-containing NMDARs.

\section{ABA induction augments postsynaptic NMDAR levels}

ABA induction, but not exercise, increased both NR2A and NR2B at postsynaptic membranes (Fig. 3). Do NR2A and NR2B immunoreactivity reflect functional NMDARs sites? While earlier biochemical studies have indicated that NR2 subunits are not transported to the cell surface unless associated with NR1 subunits (Reviewed in (McIlhinney et al. 2003)), a more recent study using fluorophores tagged to NR1 and NR2 subunits and total internal reflection fluorescent microscopy to restrict illumination to the plasma membrane indicated that as much as a third of the NR2 subunits at the plasma membrane of Xenopus oocytes could be without NR1 (Ulbrich and Isacoff 2008). Thus, we presume that the majority of NR2A and NR2B subunits at the plasma membrane reflect NR2A and NR2Bcontaining functional NMDARs. Compared to the ABA groups, the increase of NR2A was less for the group that experienced food restriction alone (Fig. 3a), as were the increases of NR2B for the groups that experienced food restriction alone and exercise alone (Fig. 3b), with none of these differences reaching statistical significance. These changes, elicited most strongly by ABA induction, would likely increase excitability of pyramidal cells. This effect could add to the increased neuronal excitability associated with dendritic retraction (Chowdhury et al. 2014a), which could increase neurons' input resistance.

NR2B immunoreactivity was also increased at the presynaptic membrane by ABA induction and food restriction alone (Fig. 3b). Presynaptic NR2B-NMDARs tonically facilitate 
glutamate release (Li et al. 2009; Yang et al. 2006), increasing hippocampal excitability additively with the postsynaptic mechanisms. The rise of pre- and postsynaptic NMDARs could facilitate LTP (long-term potentiation), learning, and memory, often associated more with the rise of NR2A-NMDARs than of NR2B-NMDARs (Liu et al. 2004; Massey et al. 2004). Supporting this prediction, other cohorts of adolescent female rats that underwent identical $\mathrm{ABA}$ and food-restriction-alone treatments exhibited faster acquisition of spatial memory, relative to the CON animals, after recovering from ABA for a week (Aoki et al. 2016; Chowdhury et al. 2014b). Consistent with this idea, individuals diagnosed with AN are often characterized to be perfectionists, high achievers, and goal-oriented (Kaye et al. 2009; Bachner-Melman et al. 2007; Sundquist et al. 2016; Dura and Bornstein 1989; Beals 2004).

Although exercise alone did not alter postsynaptic levels of NMDARs, extra-synaptic spine membrane NR2A immunoreactivity was elevated by exercise. Unlike the excitotoxic and apoptotic roles ascribed to extra-synaptic NR2B-NMDARs expressed by cultured neurons (Hardingham and Bading 2010; Kaufman et al. 2012), the consequence of elevating extrasynaptic spine membrane NMDAR in vivo by exercise during adolescence may be to prune synapses, spines, and dendritic branches, as we have observed in another cohort of EX animals (given access to a running wheel without food restriction during the same adolescent ages) (Chowdhury et al. 2014a). Future studies that analyze larger group sizes of animals or after longer periods of wheel access may reveal additional changes at the postsynaptic membrane. Animals of this study were monitored for voluntary exercise only. Especially during adolescence, when exercise is experienced forcibly on a treadmill or through daily physical training, such as exposure to a rotarod over a pool of water, additional effects yielded on hippocampal CA1 spines that are detectable by electron microscopy are interesting questions for future studies. In male rats, mild food restriction for much longer durations is reported to increase life-span and delay or prevent age-related diseases (Yu et al. 1982) and cognitive decline (Pitsikas et al. 1990). Relatively less is known about the consequences of shorter term but more severe food restriction, as used in our paradigm. Our cohorts consisted of only adolescent females. Therefore, it remains to be tested whether the patterns of synaptic changes that were evoked by exercise, food restriction or of their combination is generalizable to males, other age groups or after milder but more prolonged forms of food restriction.

Spinous NMDARs relate to individual differences in ABA vulnerability: Excitability of pyramidal neurons in the dorsal hippocampus is related to the animal's stress-induced anxiety, especially for adolescent females (Shen et al. 2007). Since anxiety is positively correlated with wheel running (Wable et al. 2015b), it was surprising that neither NR2A nor NR2B immunoreactivity at postsynaptic sites correlated with the extent of wheel running. However, postsynaptic NR2B immunoreactivity did correlate with weight loss. The rise of NR2B-NMDARs has been reported to support LTD, but from extra-synaptic sites (Liu et al. 2004; Massey et al. 2004). Based on our measurements, which differentiated postsynaptic from extra-synaptic sites, we interpret the postsynaptic NR2B-containing NMDARs to be involved in increasing neuronal excitability, consistent with animals' behavior of increased anxiety and hyperactivity. 
Individual differences in ABA vulnerability (both weight change and running) correlated strongly and negatively with spines' cytoplasmic pool of NR2A-NMDARs (Fig. 4a, c); animals with more NR2A-containing NMDARs in the cytoplasm exhibited greater resilience to ABA. We propose that an active process of retention of NR2A-containing NMDARs in the cytoplasm reduces their rate of insertion into the postsynaptic region, which could be preventing over-excitability of pyramidal neurons.

Since the ABA animals' levels of cytoplasmic NR2A immunoreactivity overlap to some extent with those of the CON animals, it is possible that NR2A-containing NMDARs are tethered to the cytoplasm prior to introduction of the stressor. If so, then one prediction would be that the CON animals with relatively strong mechanisms for retention of NR2Acontaining NMDARs in the cytoplasm would be resilient, while those with weaker mechanisms would be exhibit vulnerability, should the animals become introduced to the ABA paradigm or other stressful conditions. Although we do not see this correlation of cytoplasmic NR2A immunoreactivity with weight change in the FR animals, this could be explained by an alternate reason. We have shown that animals can adapt to the restricted feeding schedule by the fourth day of food restriction, so long as the running wheel is absent (Fig. 1b): the lack of correlation may reflect a reduction in stress that comes with this adaptation, which relieves the cytoplasmic NR2A tethering mechanism.

Individual differences in wheel activity also correlated negatively with cytoplasmic NR2A in the EX group that experienced no food restriction, albeit less than what was observed for the ABA group of animals. This trend may reflect individual differences in resilience to the stress associated with single housing, since exercise is anxiogenic for singly housed animals (Fuss et al. 2010) but not for group-housed animals (Schoenfeld et al. 2013). These findings suggest that treatments targeting postsynaptic NR2B-NMDARs, such as ifenprodil (Williams 1993), may be able to put a brake on the vicious cycle of ABA that begins with elevated anxiety and leads to excessive exercise and weight loss.

The lack of correlation between postsynaptic NMDARs with wheel running may be due to the stronger influence of another neurotransmitter system. Spine membrane levels of extrasynaptic $a 4 \beta \delta$-containing $\mathrm{GABA}_{\mathrm{A}} \mathrm{Rs}$, which mediate shunting inhibition at dendritic spines of stratum radiatum of the dorsal CA1 (Shen et al. 2007), correlate strongly and negatively with the distances run by ABA animals (Aoki et al. 2014). Importantly, these a $4 \beta \delta$ $\mathrm{GABA}_{\mathrm{A}} \mathrm{Rs}$ are inactivated by allopregnanolone in a chloride ion-flux-dependent manner, a condition brought on in the dorsal CA1 of adolescent females when an animal is stressed (Shen et al. 2007). During these periods of elevated stress, transient inactivation of $a 4 \beta \delta$ $\mathrm{GABA}_{\mathrm{A}}$ Rs enables animals to fully utilize NMDARs for hippocampal LTP induction, learning and memory formation (Shen et al. 2010). As expected, exogenous progesterone, which metabolizes to allopregnanolone, exacerbates ABA vulnerability, characterized by food-restriction-evoked hyperactivity (Wable et al. 2015a). Other candidate receptors that have yet to be analyzed for their correlation with wheel running include AMPA receptors, for which no data are yet available, and serotonin 5HT4 receptors, shown to lead to anorexia-like behavior when over-expressed in the prefrontal cortex (Compan et al. 2015). This last point leads to another question - whether NMDARs or other neurotransmitters elsewhere in the brain may also be related to the animal's hyperactivity. We have shown that 
GABAergic innervation of the pre-frontal cortical pyramidal neurons is correlated with the animals' running during the days of food restriction (Chen et al. 2016).

\section{Contribution by drebrin in ABA vulnerability}

The ABA tissue exhibited higher proportion of intensely drebrin-immunolabeled PSDs than CON tissue. This group difference suggests that ABA evokes a rise of drebrin at the PSD. Although drebrin is a predominantly cytosolic protein, a portion of drebrin is found in the synaptosomal fraction that includes the PSD (personal communication with Dr. Tomoaki Shirao of Gunma University, Japan). This is not so surprising, since drebrin is an F-actin binding protein and the PSD fraction contains actin (Aoki et al. 1985).

Data from drebrin knockout brains indicate that drebrin is required for homeostatic plasticity through the NMDAR activity-dependent trafficking of NR2A-NMDARs into the spine cytoplasm (Aoki et al. 2009a). The strong negative correlation between cytoplasmic NR2A and drebrin at the PSD suggests that the relatively low levels of drebrin at the postsynaptic membrane of ABA-resilient animals could be a "bottleneck", preventing NR2A-NMDARs (but not NR2B-NMDARs, see below) from becoming readily inserted into the postsynaptic membrane, despite the elevated NR2A expression in the spine cytoplasm. The elevated levels of cytoplasmic NR2A-containing NMDARs, together with the relatively low levels of postsynaptic NR2B-NMDARs (Fig. 5f), could contribute towards dampening excitability of pyramidal neurons and the level of anxiety-evoked excessive exercise (Fig. 4c), thereby minimizing body weight loss (Fig. 4a), ultimately making the animal more resilient to ABA (Fig. 7).

Since PSD labeling for drebrin positively correlates with postsynaptic NR2B but not with postsynaptic NR2A, the ABA-evoked rise of drebrin may promote the anchoring of NR2Bcontaining NMDARs at the postsynaptic membrane, preferentially over those containing only NR2A subunits (Fig. 7). A previous study has pointed to drebrin as a candidate molecule facilitating the insertion of NR2B-NMDARs at nascent synapses, as the two molecules co-cluster in vivo in rat hippocampal neurons during early postnatal weeks (Aoki et al. 2005). However, that study did not include analysis of adolescent brain tissue. It is possible that this ABA-induced redistribution of NR2B-containing NMDARs at the postsynaptic membrane does not occur in all stages of development but does in adolescent hippocampi, also supported by findings of adolescent CA1 pyramidal cells undergoing robust dendritic re-organizations during ABA, EX, FR, and CON (Chowdhury et al. 2014a, c). Based on the observation that individuals with higher levels of postsynaptic NR2B levels lost more weight (Fig. 5b, e), we propose that the enhanced anchoring of the NR2Bcontaining NMDARs at the postsynaptic membrane via drebrin renders the animals more vulnerable to ABA induction. Drebrin's importance in synaptic plasticity and cognition during adulthood has been suggested, based on cognitive impairment related to drebrin loss in neocortex and hippocampus of animal models of Alzheimer's disease [reviewed in (Aoki et al. 2007)]. Conversely, transgenic mice with enhanced NR2B expression exhibit superior learning, memory and larger LTP (Tang et al. 2001). Furthermore, the same study has shown that environmental enrichment, which includes wheel access, increases both NR2A and NR2B protein levels in the forebrain of wild type and NR2B-transgenic mice (Tang et al. 
2001). The present EM-ICC data add to previous data based on western blots of forebrain homogenates by highlighting the important differences between the two subunits in terms of their expression pattern - postsynap-tic for NR2B-containing NMDARs versus cytoplasmic for NR2A-containing subunits, each of which can have distinct behavioral and physiological consequences, including undesirable consequences, such as increased ABA vulnerability.

Since brain-derived neurotrophic factor (BDNF) promotes the expression and trafficking of NMDARs to the plasma membrane (Caldeira et al. 2007), we propose that BDNF may be one agent translating environmentally evoked behavior to synaptic drebrin and NMDAR changes, especially since BDNF secretion is known to be elevated in the hippocampus by food restriction (Stranahan et al. 2009). BDNF secretion is strongly stimulated by estrogen (Bath et al. 2013). Therefore, one reason why vulnerability to AN peaks at puberty may be that the sudden change in gonadal hormone levels increases the responsiveness of the hippocampus to BDNF, which in turn augments neurotransmitter receptor responsiveness to chronic activity levels. Future studies that assess synaptic NMDAR levels following manipulation of gonadal hormones and BDNF secretions should help to elucidate the molecular nature of vulnerability to $\mathrm{ABA}$ and $\mathrm{AN}$, especially during adolescence.

\section{Supplementary Material}

Refer to Web version on PubMed Central for supplementary material.

\section{Acknowledgments}

We thank Gauri Wable, Alisa Liu, Clive Miranda, Jia-Yi Wang, Kei Tateyama, Ruka Aderogba and Barkha Rana for their assistance and Danielle D Mendoca for proof-reading the manuscript. This study was supported by The Klarman Foundation Grant Program in Eating Disorders Research, R21MH091445-01, R21 MH105846, R01NS066019-01A1, R01NS047557-07A1, NEI Core Grant EY13079, NYU's Research Challenge Fund, NSFREU 1460880 to CA, YWC, the Fulbright Scholarship to YWC, NYU Dean's Undergraduate Research Fund to AL and JYW, UL1 TR000038 from the National Center for the Advancement of Translational Science (NCATS) to TGC, T32 MH019524 to GSW, NYU Abu Dhabi Fund to CM and R25GM097634-01 to RA. The brain tissue used for this study is the same as that used for another study (Nedelescu et al. 2016). Therefore, a portion of the ante mortem weight and wheel running data are presented in both manuscripts.

\section{References}

Adams MM, Fink SE, Janssen WG, Shah RA, Morrison JH. Estrogen modulates synaptic $N$-methyl-Daspartate receptor subunit distribution in the aged hippocampus. J Comp Neurol. 2004; 474(3):419426. DOI: 10.1002/cne.20148 [PubMed: 15174084]

Aoki, C., Erisir, A. Experience-dependent synaptic plasticity in the developing cerebral cortex. In: Segal, M., Pickel, VM., editors. The Synapse: Structure and Function, vol 3 Neuroscience-Net Reference Book Series. 1st. Academic Press, Elsevier; 2014. p. 397-446.

Aoki C, Carlin RK, Siekevitz P. Comparison of proteins involved with cyclic AMP metabolism between synaptic membrane and postsynaptic density preparations isolated from canine cerebral cortex and cerebellum. J Neurochem. 1985; 44(3):966-978. [PubMed: 2983024]

Aoki C, Rodrigues S, Kurose H. Use of electron microscopy in the detection of adrenergic receptors. Methods Mol Biol. 2000; 126:535-563. [PubMed: 10685434]

Aoki C, Fujisawa S, Mahadomrongkul V, Shah PJ, Nader K, Erisir A. NMDA receptor blockade in intact adult cortex increases trafficking of NR2A subunits into spines, postsynaptic densities, and axon terminals. Brain Res. 2003; 963(1-2):139-149. [PubMed: 12560119]

Aoki C, Sekino Y, Hanamura K, Fujisawa S, Mahadomrongkul V, Ren Y, Shirao T. Drebrin A is a postsynaptic protein that localizes in vivo to the submembranous surface of dendritic sites forming 
excitatory synapses. J Comp Neurol. 2005; 483(4):383-402. DOI: 10.1002/cne.20449 [PubMed: 15700273]

Aoki C, Mahadomrongkul V, Fujisawa S, Habersat R, Shirao T. Chemical and morphological alterations of spines within the hippocampus and entorhinal cortex precede the onset of Alzheimer's disease pathology in double knock-in mice. J Comp Neurol. 2007; 505(4):352-362. DOI: 10.1002/ cne.21485 [PubMed: 17912741]

Aoki C, Kojima N, Sabaliauskas N, Shah L, Ahmed TH, Oakford J, Ahmed T, Yamazaki H, Hanamura K, Shirao T. Drebrin a knockout eliminates the rapid form of homeostatic synaptic plasticity at excitatory synapses of intact adult cerebral cortex. J Comp Neurol. 2009a; 517(1):105-121. [PubMed: 19711416]

Aoki C, Lee J, Nedelescu H, Ahmed T, Ho A, Shen J. Increased levels of NMDA receptor NR2A subunits at pre- and postsynaptic sites of the hippocampal CA1: An early response to conditional double knockout of presenilin 1 and 2. J Comp Neurol. 2009b; 517(4):512-523. [PubMed: 19795494]

Aoki C, Sabaliauskas N, Chowdhury T, Min JY, Colacino AR, Laurino K, Barbarich-Marsteller NC. Adolescent female rats exhibiting activity-based anorexia express elevated levels of GABA(A) receptor alpha 4 and delta subunits at the plasma membrane of hippocampal CA1 spines. Synapse. 2012; 66(5):391-407. DOI: 10.1002/syn.21528 [PubMed: 22213233]

Aoki C, Wable G, Chowdhury TG, Sabaliauskas NA, Laurino K, Barbarich-Marsteller NC. Alpha4betadelta-GABAARs in the hippocampal CA1 as a biomarker for resilience to activitybased anorexia. Neuroscience. 2014; 265:108-123. DOI: 10.1016/j.neuroscience.2014.01.011 [PubMed: 24444828]

Aoki C, Chowdhury TG, Wable GS, Chen YW. Synaptic changes in the hippocampus of adolescent female rodents associated with resilience to anxiety and suppression of food restriction-evoked hyperactivity in an animal model for anorexia nervosa. Brain Res. 2016; doi: 10.1016/j.brainres. 2016.01.019

Arcelus J, Mitchell AJ, Wales J, Nielsen S. Mortality rates in patients with anorexia nervosa and other eating disorders. A meta-analysis of 36 studies. Arch Gen Psychiatry. 2011; 68(7):724-731. DOI: 10.1001/archgenpsychiatry.2011.74 [PubMed: 21727255]

Bachner-Melman R, Lerer E, Zohar AH, Kremer I, Elizur Y, Nemanov L, Golan M, Blank S, Gritsenko I, Ebstein RP. Anorexia nervosa, perfectionism, and dopamine D4 receptor (DRD4). Am J Med Genet B Neuropsychiatr Genet. 2007; 144B(6):748-756. DOI: 10.1002/ajmg.b.30505 [PubMed: 17440932]

Barria A, Malinow R. Subunit-specific NMDA receptor trafficking to synapses. Neuron. 2002; 35(2): 345-353. [PubMed: 12160751]

Bath KG, Schilit A, Lee FS. Stress effects on BDNF expression: Effects of age, sex, and form of stress. Neuroscience. 2013; doi: 10.1016/j.neuroscience.2013.01.074

Beals KA. Disordered eating among athletes: a comprehensive guide for health professionals. Human Kinetic, Champagne. 2004

Boraska V, Franklin CS, Floyd JA, Thornton LM, Huckins LM, Southam L, Rayner NW, Tachmazidou I, Klump KL, Treasure J, Lewis CM, Schmidt U, Tozzi F, Kiezebrink K, Hebebrand J, Gorwood P, Adan RA, Kas MJ, Favaro A, Santonastaso P, Fernandez-Aranda F, Gratacos M, Rybakowski F, Dmitrzak-Weglarz M, Kaprio J, Keski-Rahkonen A, Raevuori A, Van Furth EF, Slof-Opt Landt MC, Hudson JI, Reichborn-Kjennerud T, Knudsen GP, Monteleone P, Kaplan AS, Karwautz A, Hakonarson H, Berrettini WH, Guo Y, Li D, Schork NJ, Komaki G, Ando T, Inoko H, Esko T, Fischer K, Mannik K, Metspalu A, Baker JH, Cone RD, Dackor J, Desocio JE, Hilliard CE, O'Toole JK, Pantel J, Szatkiewicz JP, Taico C, Zerwas S, Trace SE, Davis OS, Helder S, Buhren K, Burghardt R, de Zwaan M, Egberts K, Ehrlich S, Herpertz-Dahlmann B, Herzog W, Imgart H, Scherag A, Scherag S, Zipfel S, Boni C, Ramoz N, Versini A, Brandys MK, Danner UN, de Kovel C, Hendriks J, Koeleman BP, Ophoff RA, Strengman E, van Elburg AA, Bruson A, Clementi M, Degortes D, Forzan M, Tenconi E, Docampo E, Escaramis G, Jimenez-Murcia S, Lissowska J, Rajewski A, Szeszenia-Dabrowska N, Slopien A, Hauser J, Karhunen L, Meulenbelt I, Slagboom PE, Tortorella A, Maj M, Dedoussis G, Dikeos D, Gonidakis F, Tziouvas K, Tsitsika A, Papezova H, Slachtova L, Martaskova D, Kennedy JL, Levitan RD, Yilmaz Z, Huemer J, Koubek D, Merl E, Wagner G, Lichtenstein P, Breen G, Cohen-Woods S, Farmer A, McGuffin P, Cichon S, Giegling I, 
Herms S, Rujescu D, Schreiber S, Wichmann HE, Dina C, Sladek R, Gambaro G, Soranzo N, Julia A, Marsal S, Rabionet R, Gaborieau V, Dick DM, Palotie A, Ripatti S, Widen E, Andreassen OA, Espeseth T, Lundervold A, Reinvang I, Steen VM, Le Hellard S, Mattingsdal M, Ntalla I, Bencko V, Foretova L, Janout V, Navratilova M, Gallinger S, Pinto D, Scherer SW, Aschauer H, Carlberg L, Schosser A, Alfredsson L, Ding B, Klareskog L, Padyukov L, Courtet P, Guillaume S, Jaussent I, Finan C, Kalsi G, Roberts M, Logan DW, Peltonen L, Ritchie GR, Barrett JC, Estivill X, Hinney A, Sullivan PF, Collier DA, Zeggini E, Bulik CM. The Wellcome Trust Case Control C. A genome-wide association study of anorexia nervosa. Mol Psychiatry. 2014; doi: 10.1038/mp. 2013.187

Bulik CM, Thornton LM, Root TL, Pisetsky EM, Lichtenstein P, Pedersen NL. Understanding the relation between anorexia nervosa and bulimia nervosa in a Swedish national twin sample. Biol Psychiatry. 2010; 67(1):71-77. DOI: 10.1016/j.biopsych.2009.08.010 [PubMed: 19828139]

Caldeira MV, Melo CV, Pereira DB, Carvalho RF, Carvalho AL, Duarte CB. BDNF regulates the expression and traffic of NMDA receptors in cultured hippocampal neurons. Mol Cell Neurosci. 2007; 35(2):208-219. DOI: 10.1016/j.mcn.2007.02.019 [PubMed: 17428676]

Casper RC, Sullivan EL, Tecott L. Relevance of animal models to human eating disorders and obesity. Psychopharmacol. 2008; 199(3):313-329.

Chen YW, Wable GS, Chowdhury TG, Aoki C. Enlargement of axo-somatic contacts formed by GADimmunoreactive axon terminals onto layer $\mathrm{V}$ pyramidal neurons in the medial prefrontal cortex of adolescent female mice is associated with suppression of food restriction-evoked hyperactivity and resilience to activity-based anorexia. Cereb Cortex. 2016; 26(6):2574-2589. DOI: 10.1093/cercor/ bhv087 [PubMed: 25979087]

Chowdhury TG, Wable GS, Sabaliauskas NA, Aoki C. Adolescent female C57BL/6 mice with vulnerability to activity-based anorexia exhibit weak inhibitory input onto hippocam-pal CA1 pyramidal cells. Neuroscience. 2013; 241:250-267. DOI: 10.1016/j.neuroscience.2013.03.020 [PubMed: 23523748]

Chowdhury TG, Barbarich-Marsteller NC, Chan TE, Aoki C. Activity-based anorexia has differential effects on apical dendritic branching in dorsal and ventral hippocampal CA1. Brain Struct Funct. 2014a; 219(6):1935-1945. DOI: 10.1007/s00429-013-0612-9 [PubMed: 23959245]

Chowdhury TG, Rios MB, Chan TE, Cassataro DS, Barbarich-Marsteller NC, Aoki C. Activity-based anorexia during adolescence disrupts normal development of the CA1 pyramidal cells in the ventral hippocampus of female rats. Hippocampus. 2014b; 24(12):1421-1429. DOI: 10.1002/hipo. 22320 [PubMed: 24976385]

Chowdhury, TG., Fenton, AA., Aoki, C. Adolescent experience of food restriction results in delayed enhancement of spatial learning in female rats. Paper presented at the Annual Meeting of the Society for Neuroscience; Washington, D.C. 2014b.

Compan V, Walsh BT, Kaye W, Geliebter A. How does the brain implement adaptive decision making to eat? J Neurosci. 2015; 35(41):13868-13878. DOI: 10.1523/JNEUROSCI.2602-15.2015 [PubMed: 26468187]

Corson J, Nahmani M, Lubarsky K, Badr N, Wright C, Erisir A. Sensory activity differentially modulates $\mathrm{N}$-methyl-D-aspartate receptor subunits $2 \mathrm{~A}$ and $2 \mathrm{~B}$ in cortical layers. Neuroscience. 2009; 163(3):920-932. DOI: 10.1016/j.neuroscience.2009.07.016 [PubMed: 19596055]

Davis C, Katzman DK, Kaptein S, Kirsh C, Brewer H, Kalmbach K, Olmsted MP, Woodside DB, Kaplan AS. The prevalence of high-level exercise in the eating disorders: etiological implications. Compr Psychiatry. 1997; 38(6):321-326. [PubMed: 9406737]

Dura JR, Bornstein RA. Differences between IQ and school achievement in anorexia nervosa. J Clin Psychol. 1989; 45(3):433-435. [PubMed: 2745732]

Fujisawa $\mathrm{S}$, Aoki C. In vivo blockade of $\mathrm{N}$-methyl-D-aspartate receptors induces rapid trafficking of NR2B subunits away from synapses and out of spines and terminals in adult cortex. Neuroscience. 2003; 121(1):51-63. [PubMed: 12946699]

Fuss J, Ben Abdallah NM, Vogt MA, Touma C, Pacifici PG, Palme R, Witzemann V, Hellweg R, Gass P. Voluntary exercise induces anxiety-like behavior in adult C57BL/6 J mice correlating with hippocampal neurogenesis. Hippocampus. 2010; 20(3):364-376. DOI: 10.1002/hipo.20634 [PubMed: 19452518] 
Greer PL, Greenberg ME. From synapse to nucleus: calcium-dependent gene transcription in the control of synapse development and function. Neuron. 2008; 59(6):846-860. DOI: 10.1016/ j.neuron.2008.09.002 [PubMed: 18817726]

Guisinger S. Adapted to flee famine: adding an evolutionary perspective on anorexia nervosa. Psychol Rev. 2003; 110(4):745-761. [PubMed: 14599241]

Gutierrez E. A rat in the labyrinth of anorexia nervosa: contributions of the activity-based anorexia rodent model to the understanding of anorexia nervosa. Int J Eat Disord. 2013; 46(4):289-301. [PubMed: 23354987]

Hardingham GE, Bading H. Synaptic versus extrasynaptic NMDA receptor signalling: implications for neurodegenerative disorders. Nat Rev Neurosci. 2010; 11(10):682-696. DOI: 10.1038/nrn2911 [PubMed: 20842175]

Hensch TK, Fagiolini M. Excitatory-inhibitory balance and critical period plasticity in developing visual cortex. Prog Brain Res. 2005; 147:115-124. DOI: 10.1016/S0079-6123(04)47009-5 [PubMed: 15581701]

Hudson JI, Hiripi E, Pope HG Jr, Kessler RC. The prevalence and correlates of eating disorders in the national comorbidity survey replication. Biol Psychiatry. 2007; 61(3):348-358. DOI: 10.1016/ j.biopsych.2006.03.040 [PubMed: 16815322]

Kaufman AM, Milnerwood AJ, Sepers MD, Coquinco A, She K, Wang L, Lee H, Craig AM, Cynader M, Raymond LA. Opposing roles of synaptic and extrasynaptic NMDA receptor signaling in cocultured striatal and cortical neurons. J Neurosci. 2012; 32(12):3992-4003. DOI: 10.1523/ JNEUROSCI.4129-11.2012 [PubMed: 22442066]

Kaye WH, Bulik CM, Thornton L, Barbarich N, Masters K. Comorbidity of anxiety disorders with anorexia and bulimia nervosa. Am J Psychiatry. 2004; 161(12):2215-2221. [PubMed: 15569892]

Kaye WH, Fudge JL, Paulus M. New insights into symptoms and neurocircuit function of anorexia nervosa. Nat Rev Neurosci. 2009; 10(8):573-584. DOI: 10.1038/nrn2682 [PubMed: 19603056]

Klump KL, Miller KB, Keel PK, McGue M, Iacono WG. Genetic and environmental influences on anorexia nervosa syndromes in a population-based twin sample. Psychol Med. 2001; 31(4):737740. [PubMed: 11352375]

Kron L, Katz JL, Gorzynski G, Weiner H. Hyperactivity in anorexia nervosa: a fundamental clinical feature. Compr Psychiatry. 1978; 19(5):433-440. [PubMed: 679677]

Li YH, Wang J, Zhang G. Presynaptic NR2B-containing NMDA autoreceptors mediate gluta-matergic synaptic transmission in the rat visual cortex. Curr Neurovasc Res. 2009; 6(2):104-109. [PubMed: 19442159]

Liu L, Wong TP, Pozza MF, Lingenhoehl K, Wang Y, Sheng M, Auberson YP, Wang YT. Role of NMDA receptor subtypes in governing the direction of hippocampal synaptic plasticity. Science. 2004; 304(5673):1021-1024. DOI: 10.1126/science.1096615 [PubMed: 15143284]

Martin PD, Berthoz A. Development of spatial firing in the hippocampus of young rats. Hippocampus. 2002; 12(4):465-480. DOI: 10.1002/hipo.10021 [PubMed: 12201631]

Massey PV, Johnson BE, Moult PR, Auberson YP, Brown MW, Molnar E, Collingridge GL, Bashir ZI. Differential roles of NR2A and NR2B-containing NMDA receptors in cortical long-term potentiation and long-term depression. J Neurosci. 2004; 24(36):7821-7828. DOI: 10.1523/ JNEUROSCI.1697-04.2004 [PubMed: 15356193]

McIlhinney RA, Philipps E, Le Bourdelles B, Grimwood S, Wafford K, Sandhu S, Whiting P. Assembly of $N$-methyl-D-aspartate (NMDA) receptors. Biochem Soc Trans. 2003; 31(Pt 4):865868. [PubMed: 12887323]

Meijer JH, Robbers Y. Wheel running in the wild. Proc Bio Sci/R Soc. 2014; doi: 10.1098/rspb. 2014.0210

Nedelescu H, Chowdhury TG, Wable GS, Arbuthnott G, Aoki C. Cerebellar sub-divisions differ in exercise-induced plasticity of noradrenergic axons and in their association with resilience to activity-based anorexia. Brain Struct Funct. 2016; doi: 10.1007/s00429-016-1220-2

Peters, A., Palay, SL., Webster, Hd. The fine structure of the nervous system: neurons and their supporting cells. 3rd. Oxford University; New York: 1991. 
Petralia RS, Wang YX, Hua F, Yi Z, Zhou A, Ge L, Stephenson FA, Wenthold RJ. Organization of NMDA receptors at extrasynaptic locations. Neuroscience. 2010; 167(1):68-87. DOI: 10.1016/ j.neuroscience.2010.01.022 [PubMed: 20096331]

Phend KD, Rustioni A, Weinberg RJ. An osmium-free method of epon embedment that preserves both ultrastructure and antigenicity for post-embedding immunocytochemistry. J His-tochem Cytochem. 1995; 43(3):283-292.

Pitsikas N, Carli M, Fidecka S, Algeri S. Effect of life-long hypocaloric diet on age-related changes in motor and cognitive behavior in a rat population. Neurobiol Aging. 1990; 11(4):417-423. [PubMed: 2381501]

Rinaldi T, Kulangara K, Antoniello K, Markram H. Elevated NMDA receptor levels and enhanced postsynaptic long-term potentiation induced by prenatal exposure to valproic acid. Proc Natl Acad Sci USA. 2007; 104(33):13501-13506. DOI: 10.1073/pnas.0704391104 [PubMed: 17675408]

Schoenfeld TJ, Rada P, Pieruzzini PR, Hsueh B, Gould E. Physical exercise prevents stress-induced activation of granule neurons and enhances local inhibitory mechanisms in the dentate gyrus. $\mathrm{J}$ Neurosci. 2013; 33(18):7770-7777. DOI: 10.1523/JNEUROSCI.5352-12.2013 [PubMed: 23637169]

Shen H, Gong QH, Aoki C, Yuan M, Ruderman Y, Dattilo M, Williams K, Smith SS. Reversal of neurosteroid effects at alpha4beta2delta GABAA receptors triggers anxiety at puberty. Nat Neurosci. 2007; 10(4):469-477. [PubMed: 17351635]

Shen H, Sabaliauskas N, Sherpa A, Fenton AA, Stelzer A, Aoki C, Smith SS. A critical role for alpha4betadelta GABAA receptors in shaping learning deficits at puberty in mice. Science. 2010; 327(5972):1515-1518. [PubMed: 20299596]

Steinhausen HC. The outcome of anorexia nervosa in the 20th century. Am J Psychiatry. 2002; 159(8): 1284-1293. [PubMed: 12153817]

Stranahan AM, Lee K, Martin B, Maudsley S, Golden E, Cutler RG, Mattson MP. Voluntary exercise and caloric restriction enhance hippocampal dendritic spine density and BDNF levels in diabetic mice. Hippocampus. 2009; 19(10):951-961. DOI: 10.1002/hipo.20577 [PubMed: 19280661]

Sullivan PF. Mortality in anorexia nervosa. Am J Psychiatry. 1995; 152(7):1073-1074. [PubMed: 7793446]

Sundquist J, Ohlsson H, Winkleby MA, Sundquist K, Crump C. School achievement and risk of eating disorders in a Swedish National cohort. J Am Acad Child Adolesc Psychiatry. 2016; 55(1):41-46 e41. DOI: 10.1016/j.jaac.2015.09.021 [PubMed: 26703908]

Tang YP, Wang H, Feng R, Kyin M, Tsien JZ. Differential effects of enrichment on learning and memory function in NR2B transgenic mice. Neuropharmacology. 2001; 41(6):779-790. [PubMed: 11640933]

Thomas CG, Miller AJ, Westbrook GL. Synaptic and extrasynaptic NMDA receptor NR2 subunits in cultured hippocampal neurons. J Neurophysiol. 2006; 95(3):1727-1734. DOI: 10.1152/jn. 00771.2005 [PubMed: 16319212]

Tovar KR, Westbrook GL. The incorporation of NMDA receptors with a distinct subunit composition at nascent hippocampal synapses in vitro. J Neurosci. 1999; 19(10):4180-4188. [PubMed: 10234045]

Ulbrich MH, Isacoff EY. Rules of engagement for NMDA receptor subunits. Proc Natl Acad Sci USA. 2008; 105(37):14163-14168. DOI: 10.1073/pnas.0802075105 [PubMed: 18779583]

Wable GS, Chen YW, Rashid S, Aoki C. Exogenous progesterone exacerbates running response of adolescent female mice to repeated food restriction stress by changing alpha4-GABAA receptor activity of hippocampal pyramidal cells. Neuroscience. 2015a; 310:322-341. DOI: 10.1016/ j.neuroscience.2015.09.006 [PubMed: 26383252]

Wable GS, Min JY, Chen YW, Aoki C. Anxiety is correlated with running in adolescent female mice undergoing activity-based anorexia. Behav Neurosci. 2015b; 129(2):170-182. DOI: 10.1037/ bne0000040 [PubMed: 25730124]

Wade TD, Bulik CM, Neale M, Kendler KS. Anorexia nervosa and major depression: shared genetic and environmental risk factors. Am J Psychiatry. 2000; 157(3):469-471. [PubMed: 10698830] 
Williams K. Ifenprodil discriminates subtypes of the $N$-methyl-D-aspartate receptor: selectivity and mechanisms at recombinant heteromeric receptors. Mol Pharmacol. 1993; 44(4):851-859. [PubMed: 7901753]

Yang J, Woodhall GL, Jones RS. Tonic facilitation of glutamate release by presynaptic NR2Bcontaining NMDA receptors is increased in the entorhinal cortex of chronically epileptic rats. $\mathrm{J}$ Neurosci. 2006; 26(2):406-410. DOI: 10.1523/JNEUROSCI.4413-05.2006 [PubMed: 16407536]

Yu BP, Masoro EJ, Murata I, Bertrand HA, Lynd FT. Life span study of SPF Fischer 344 male rats fed ad libitum or restricted diets: longevity, growth, lean body mass and disease. J Gerontol. 1982; 37(2):130-141. [PubMed: 7056998] 
A

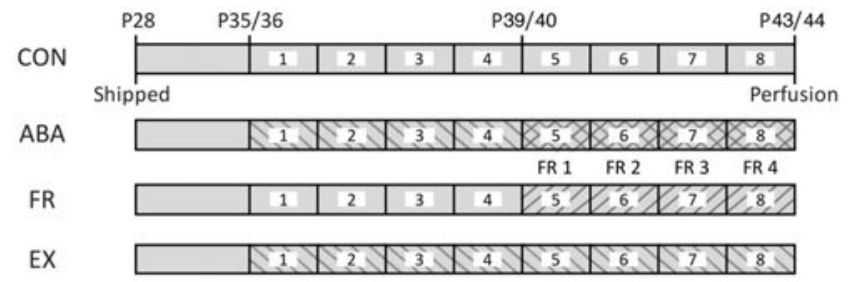

Singly housed

Ad lib food and wheel
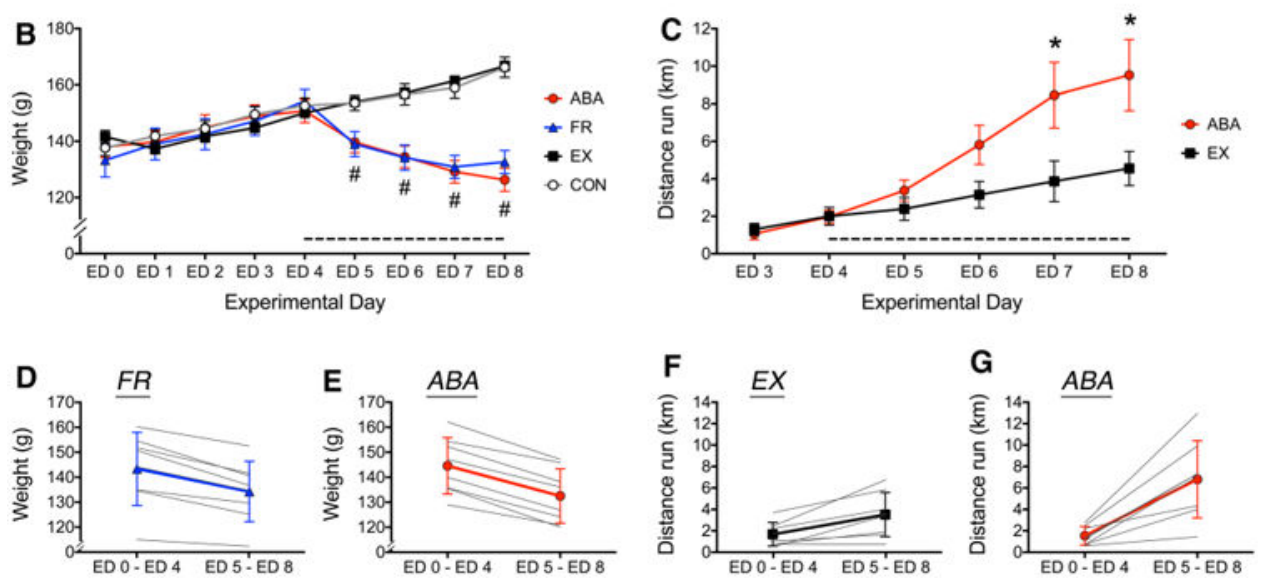

Fig. 1.

Body weight and wheel activity of CON, ABA, FR, and EX groups. a Timeline of the environmental conditions of the four experimental groups. Animals were food restricted (FR), given ad libitum access to a running wheel to allow for voluntary exercise (EX), induced to undergo activity-based anorexia (ABA) by combining wheel access and food restriction $(\mathrm{ABA})$, or served as controls $(\mathrm{CON})$ that neither exercised nor were food restricted. All animals were shipped to NYU's animal facility at postnatal day (P) 28. Wheel access for the ABA and the EX group began on experimental day 1 when animals were P35 or 36. Food restriction for the ABA and the FR groups began on experimental day 1 (FR1), which corresponded to the fifth experimental day when animals were P39 or 40. All animals were euthanized by transcardial perfusion under anesthesia on P43/44. b Body weights of all groups are shown from ED1 through ED8, as mean \pm SEM. Hash indicates a main effect of food restriction with $p<0.05$. There was no significant interaction between the effect of food restriction and wheel access. The dashed line along the $\mathrm{x}$-axis indicates the foodrestricted period, beginning on ED4. c Average voluntary running wheel activity is displayed for the EX and ABA groups for the last 6 of the 8 days of wheel access. Asterisk indicates significant difference between EX and ABA at $p<0.05$. d, e Average weight of the FR and ABA groups across the first four experimental days (ED1 through ED4) is compared to the average weight across the last four food-restricted days (ED5 through ED8). Each gray line represents data from a single animal, while the heavier line represents the group average as mean \pm SD. f, $\mathbf{g}$ The average running activity per $24 \mathrm{~h}$ during the first 4 days of wheel access was compared to the average activity during the last 4 days of wheel access in the EX and the ABA groups. Each gray line represents a single subject, while the heavier lines represent 
the group average as mean $\pm \mathrm{SD}$. In the ABA group, the latter 4 days of wheel access were accompanied by food restriction. 1 wheel count equaled $0.64 \mathrm{~m}$ 


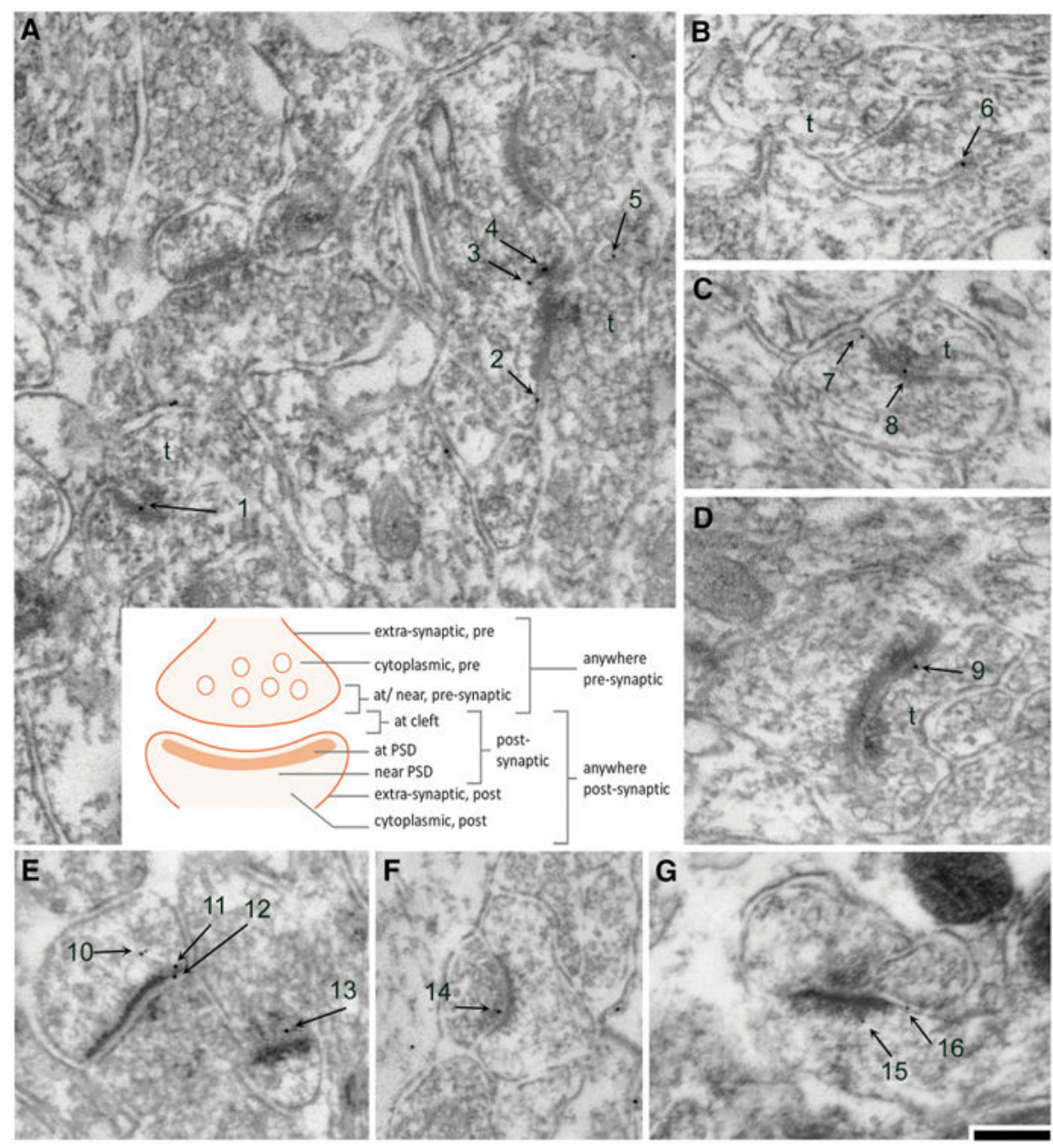

Fig. 2.

Categorization of subcellular sites in the vicinity of axo-spinous asymmetric synapses where NR2A and NR2B immunoreactivities were observed. a through e are examples of electron micrographs taken from tissue immunolabeled for the NR2A subunits, while $\mathbf{f}, \mathbf{g}$ are examples of micrographs taken from tissue immunolabeled for the NR2B subunits. a-d, $\mathbf{f}$ are micrographs taken from EX tissue, while e, g were taken from ABA tissue. The numbers depict PEG particles associated with axo-spinous asymmetric synapses. The presynaptic sides are indicated by $\mathrm{t}$ for axon terminal. Calibration bar is equal to $200 \mathrm{~nm}$ and applies to all panels. The cartoon in the center describes the categorization of subcellular location of PEG particles. The subcellular position for each particle in these examples were categorized as follows: 1 at PSD; 2 at extra-synaptic spine plasma membrane, 3 and 4 near PSD, 5 as cytoplasmic on the presynaptic side, 6 and 7 at extra-synaptic spine plasma membrane, 8 at cleft, 9 at/near presynaptic plasma membrane, 10 (two particles) as cytoplasmic within the postsynaptic spine, 11 near PSD, 12 at cleft, 13 at/near presynaptic axon terminal, 14 at/near presynaptic plasma membrane, 15 near PSD, and 16 at extra-synaptic spine plasma membrane 
A
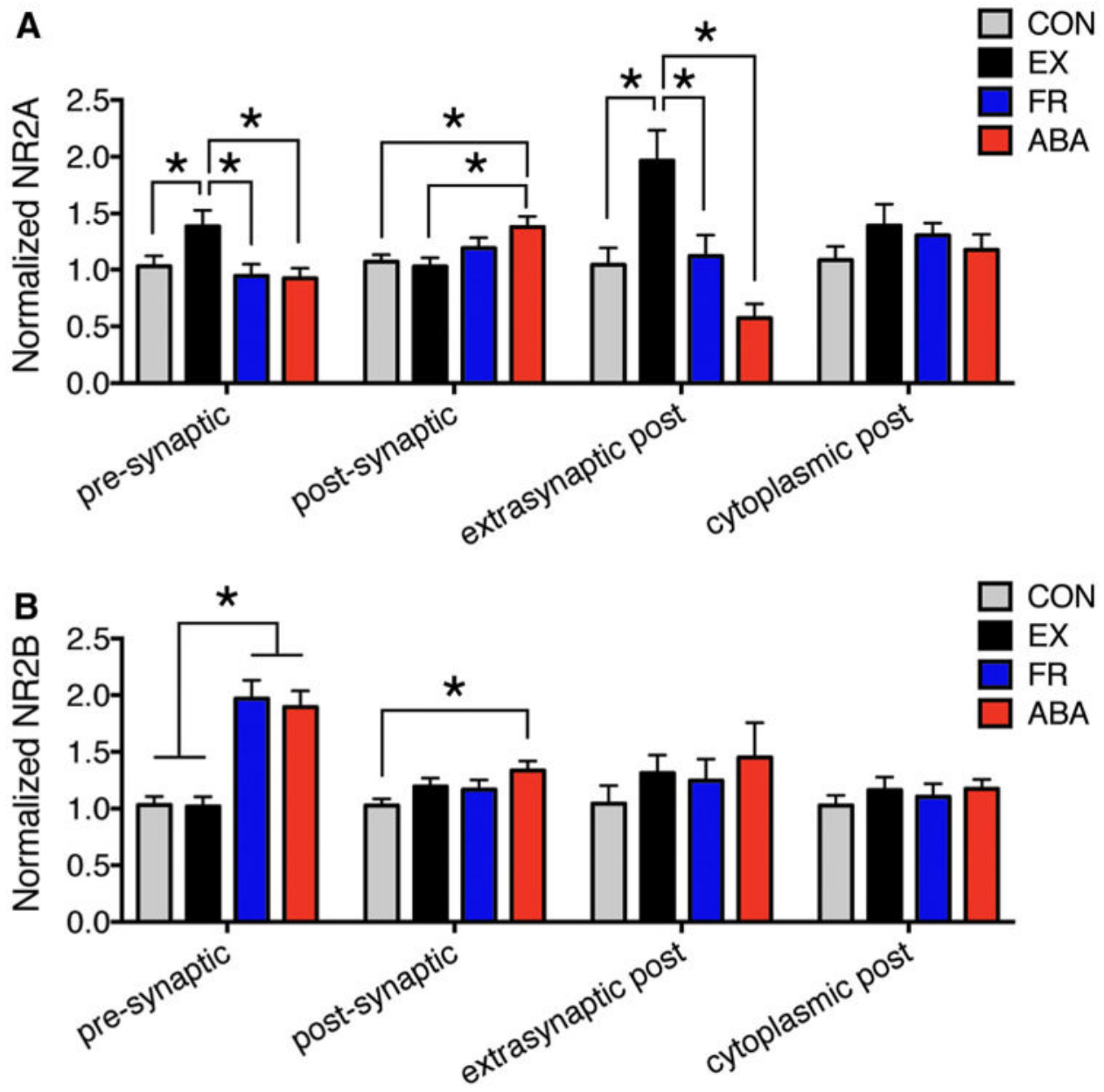

Fig. 3.

ABA elevates both NR2A and NR2B immunoreactivity specifically at the postsynaptic membrane. The histograms depict group comparisons of the number of PEG particles encountered per-10 synapses, at and near axo-spinous junctions, reflecting NR2A (a) and NR2B (b) immunoreactivitynormalized to the average of the CON values. Asterisk depicts significance of difference at $p<0.05$ by two-way ANOVA, followed by Fisher's LSD post hoc analysis. The original, pre-normalized CON values for NR2A and NR2B immunoreactivity are shown in Table 2 

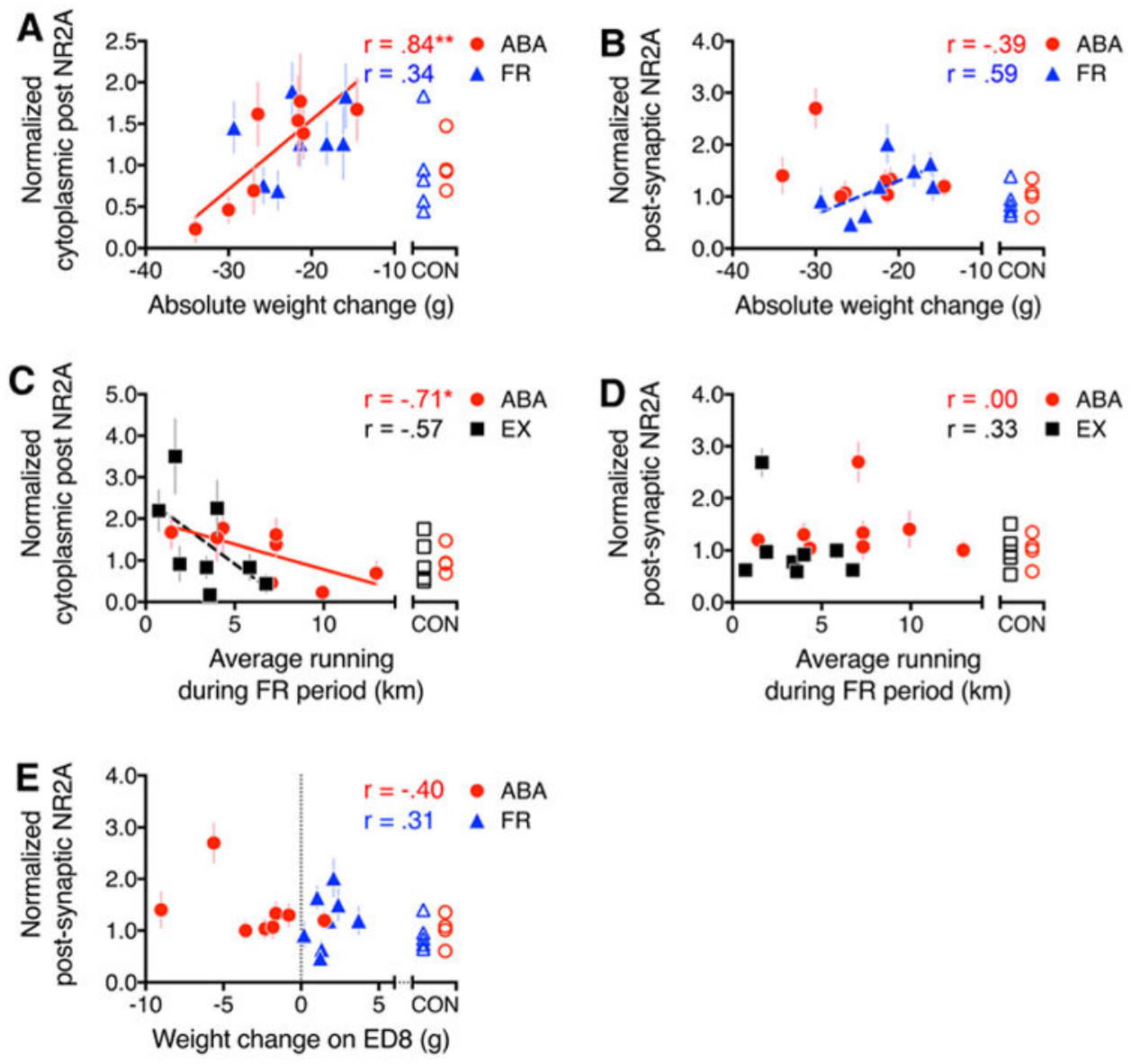

Fig. 4.

Correlations between ABA vulnerability and NR2A immunoreactivity. Pearson correlation analyses were run to determine the relationship between ABA vulnerability and the level of NR2A-PEG particle labeling. a, b ABA vulnerability was measured as the extent of body weight change, following 4 days of food restriction. c, $\mathbf{d}$ ABA vulnerability was measured as the average daily running during the 4 days of food restriction. e ABA vulnerability was measured as the weight change during the last day of food restriction, on ED 8. a, c Significant correlations between ABA vulnerability and NR2A immunoreactivity within the spine cytoplasm. b, d, e Lack of correlation between postsynaptic NR2A and ABA vulnerability. All values were normalized to a single value derived from an average of all CON tissue that were immunolabeled by the PEG procedure in parallel with the experimental group (ABA, FR, or EX). The shape of the unfilled symbols indicates the experimental group with which the $\mathrm{CON}$ tissue runs. $\mathrm{R}$ values of the Pearson correlation are indicated for each graph. Asterisk indicates $p<0.05$, and double asterisk indicates $p<0.01$. Solid lines represent trend lines for correlations with $p<0.05$, while dashed lines represent trend lines for correlations with $p>0.05$ but $₫ 0.1$ 

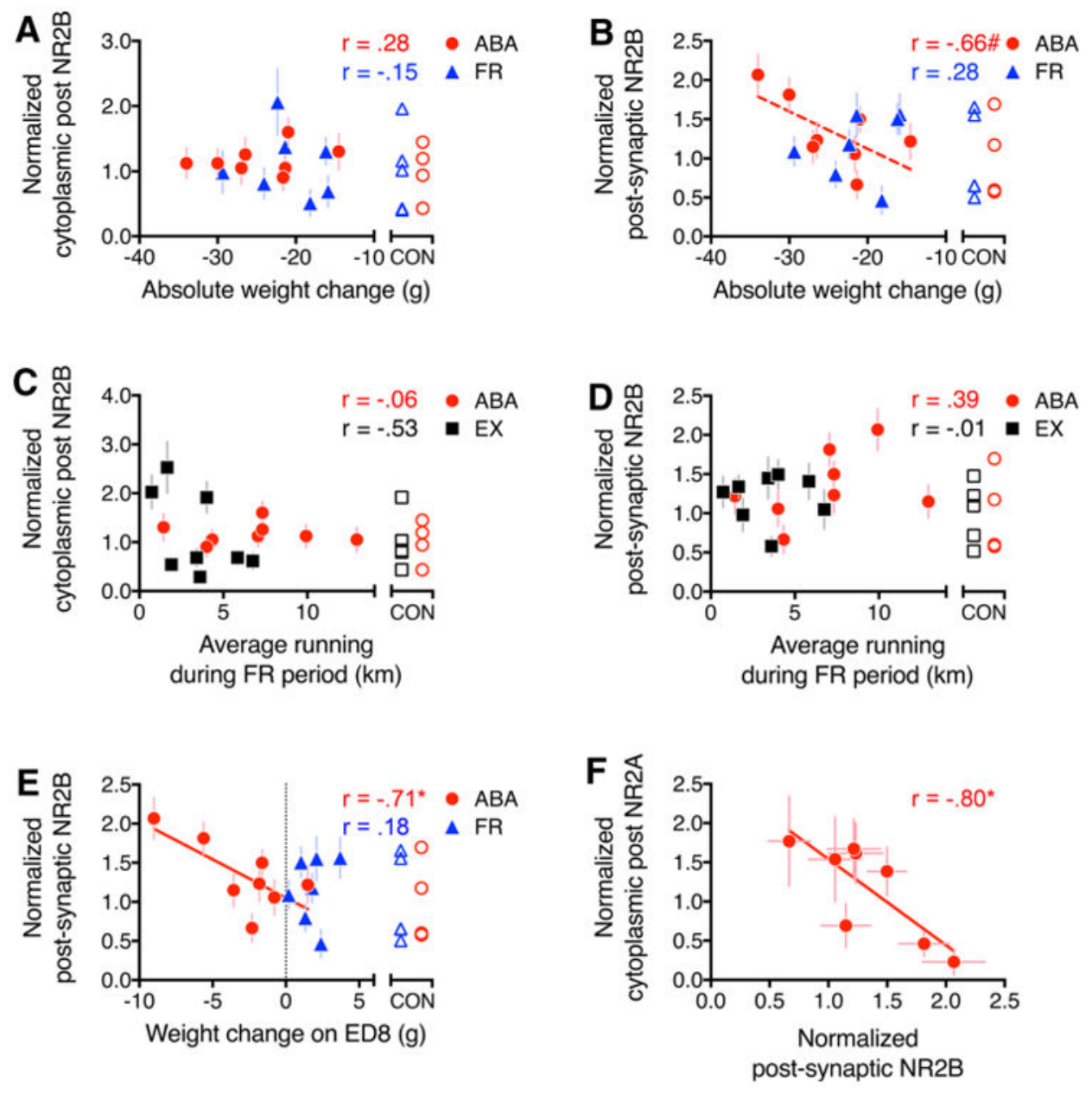

Fig. 5.

Correlations between ABA vulnerability and NR2B immunoreactivity. Pearson correlation analyses were run to determine the relationship between $A B A$ vulnerability and the level of NR2B-PEG particle labeling, just as was conducted for NR2A immunolabeling. a, b ABA vulnerability was measured as the extent of body weight change during the four days of food restriction. $\mathbf{c}, \mathbf{d} \mathrm{ABA}$ vulnerability was measured as the average daily running during the four days of food restriction. e ABA vulnerability was measured as the weight change during the last day of food restriction, on ED 8. The only measure of vulnerability that shows correlation to NR2B immunoreactivity is the extent of weight change on ED 8 (e). All values were normalized to a single value derived from an average of all CON tissue that were immunolabeled by the PEG procedure in parallel with the experimental group (ABA, FR or EX). The shape of the unfilled symbols indicates the experimental group with which the CON tissue runs. f Outcome of the Pearson correlation analysis comparing normalized cytoplasmic NR2A to normalized postsynaptic NR2B. R values of the Pearson correlation are indicated for each graph. Asterisk indicates $p<0.05$; solid lines represent trend lines for correlations with $p<0.05$; and hash and dashed lines indicate $p>0.05$ but $₫) .1$ 

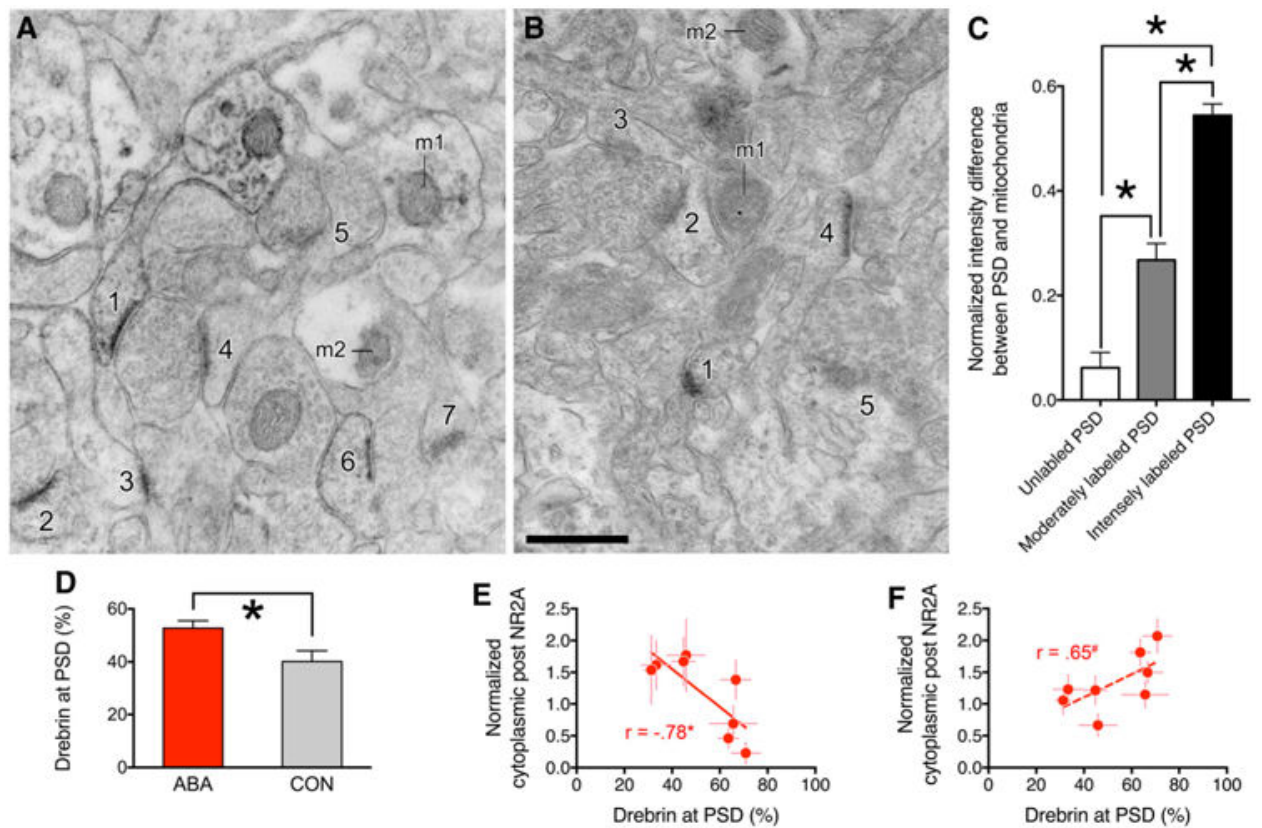

Fig. 6.

Correlations between drebrin at PSDs and NMDAR subunits at locations associated with ABA vulnerability. a (from an ABA animal) and $\mathbf{b}$ (from a CON animal) show examples of drebrin immunoreactivity at the PSD. The mitochondrial profile is indicated by $m 1$ and $m 2$ in the panels. The levels of drebrin immnuoreactivity were categorized to be the following for the 12 PSDs shown in the two panels. a 1,2,3, and 6 are intensely labeled, 4 is moderately labeled, and 5 and 7 are unlabeled. b 1 is intensely labeled, 4 is moderately labeled, and 2, 3 and 5 are unlabeled. Calibration bar equals $500 \mathrm{~nm}$ and applies to both panels. c shows intensity (gray value) difference between PSD and mitochondria normalized to the gray value of the mitochondria for the three categories of drebrin-immunolabeled PSDs. Mean gray values were sampled from 11 micrographs of ABA tissue (from 3 ABA animals) and 10 micrographs of CON tissue (from $3 \mathrm{CON}$ animals). Mean gray values of PSDs categorized as intensely labeled were significantly greater than values of PSDs categorized as unlabeled or moderately labeled. Asterisk indicates significance $(p<0.05)$. d Quantification of the proportion of intensely drebrin-immunolabeled PSDs for ABA and CON groups. 768 and 384 spines were sampled from eight ABA and four CON tissues, respectively. e Normalized cytoplasmic NR2A labeling levels of the 8 ABA animals (the same as the values shown in Fig. 4a and c) are negatively correlated to the proportion of spines immunolabeled intensely over the PSD. This correlation is significant $(p<0.05)$. $\mathbf{f}$ shows that normalized postsynaptic NR2B labeling (the same as those shown in Fig. $5 \mathrm{~b}$ and e) of the eight ABA animals is related to the proportion of spines immunolabeled intensely over the PSD. This correlation approached significance $(p=0.08)$, and is indicated by a dashed line 


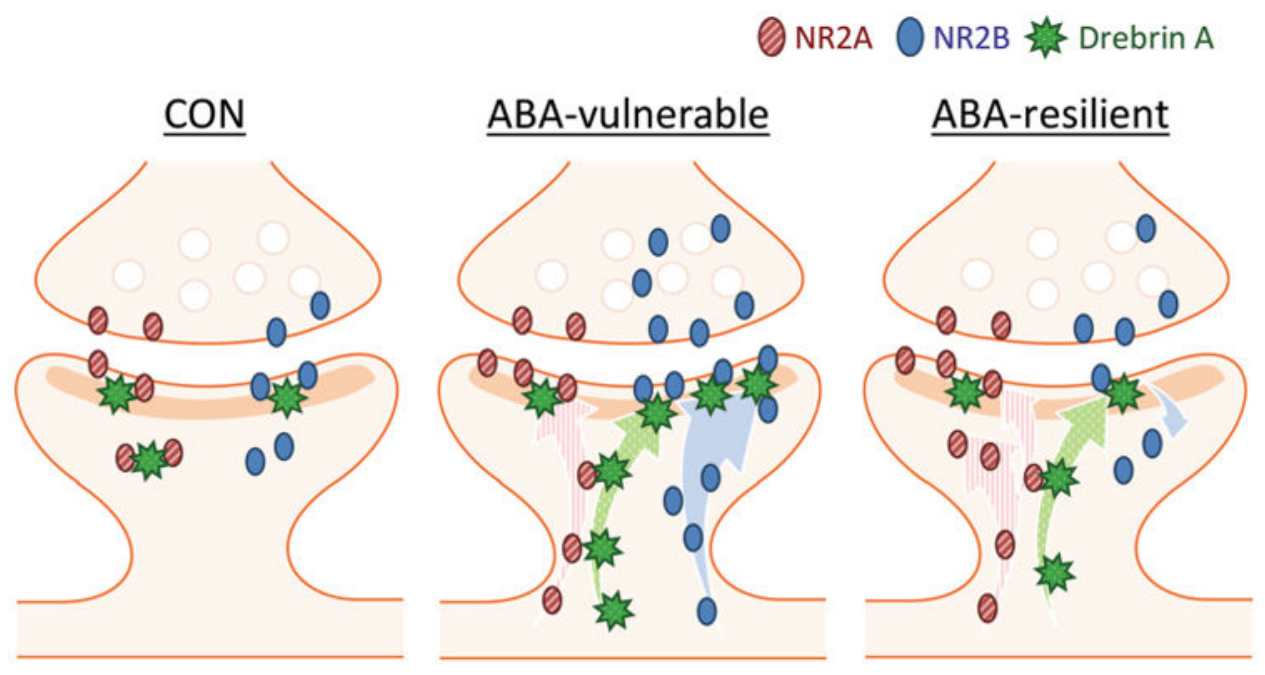

Fig. 7.

Summary of the levels of NR2A-containing NMDARs, NR2B-containing NMDARs, and drebrin at postsynaptic versus cytoplasmic locations reported for spines belonging to hippocampi of ABA-vulnerable and ABA-resilient individuals. The strongly correlating pools of synaptic proteins are shown as putatively interacting through direct contacts. The correlations suggest that the inactive pool of cytoplasmic NR2A level is actively retained from becoming inserted into the PSD through interaction with drebrin, and that drebrin interacts relatively more strongly with NR2B-containing NMDARs at the PSD than with NR2A-containing NMDARs 
Table 1

Number of synapses and animals used for NR2A and NR2B EM analysis for each experimental and control group

\begin{tabular}{lll}
\hline & NR2A & NR2B \\
\hline ABA-CON comparison & 8 ABA (1370 synapses) & 8 ABA (1580 synapses) \\
& 4 CON (680 synapses) & 4 CON (790 synapses) \\
EX-CON comparison & 8 EX (1580 synapses) & 8 EX (1590 synapses) \\
& 5 CON (1000 synapses) & 5 CON (990 synapses) \\
FR-CON comparison & 8 FR (1590 synapses) & 7 FR (1360 synapses) \\
& 5 CON (1000 synapses) & 5 CON (980 synapses) \\
\hline
\end{tabular}




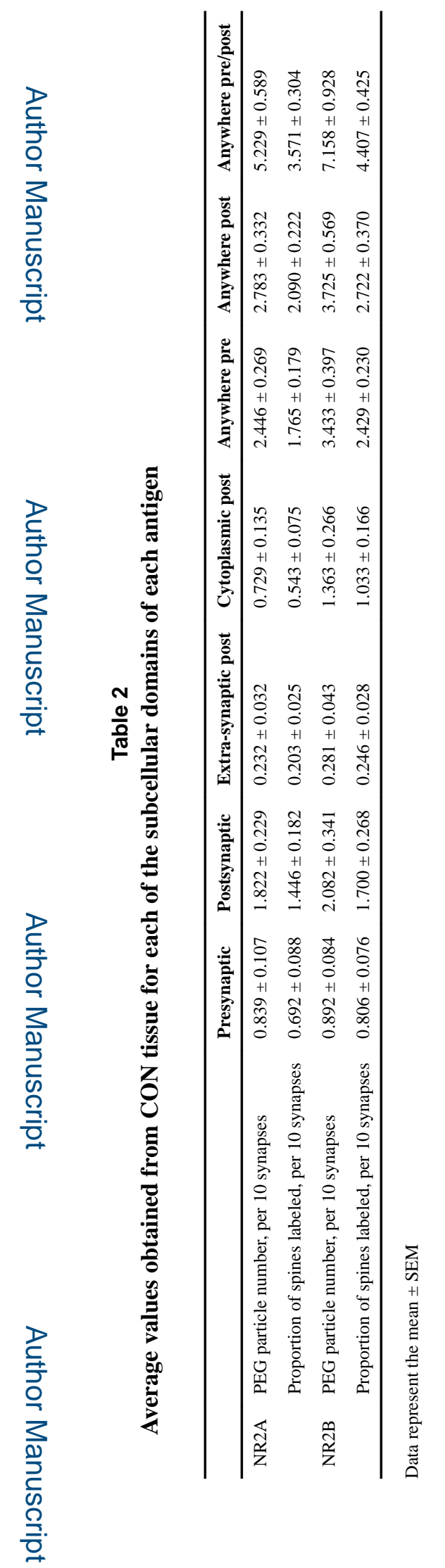

Brain Struct Funct. Author manuscript; available in PMC 2018 July 01. 


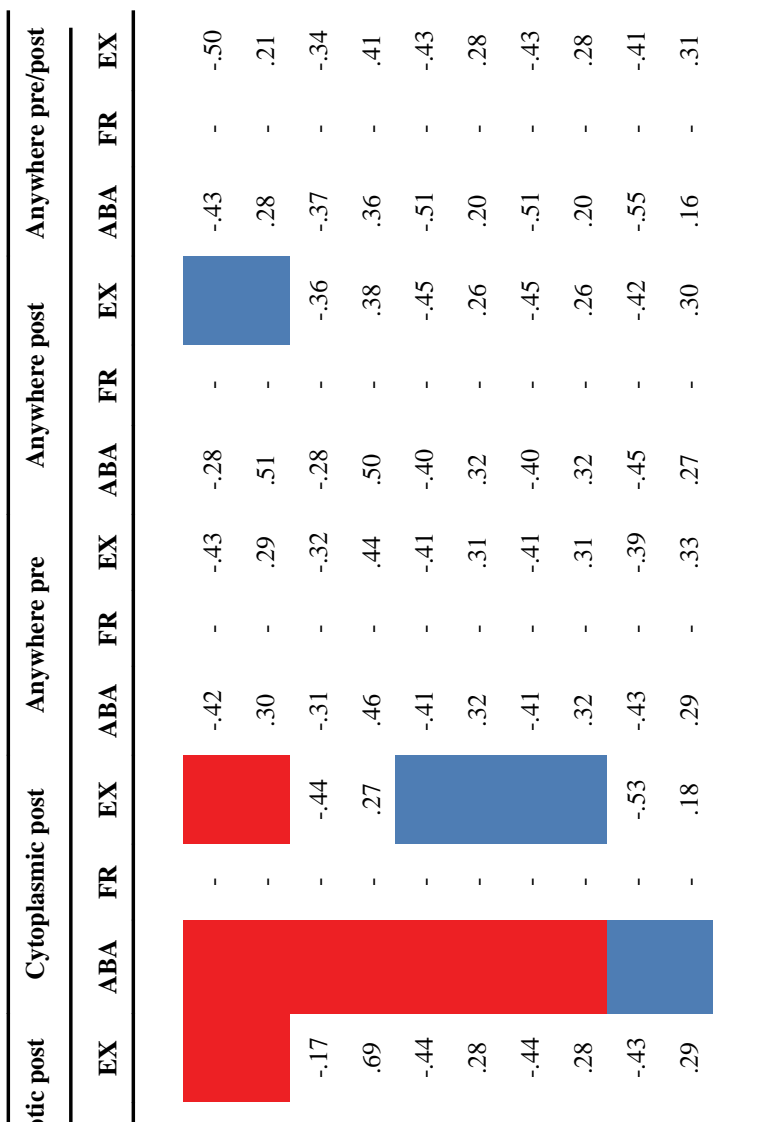

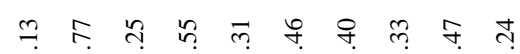

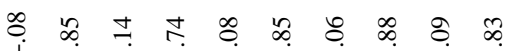
กิ $\stackrel{\infty}{?}$

竞

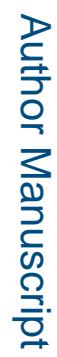
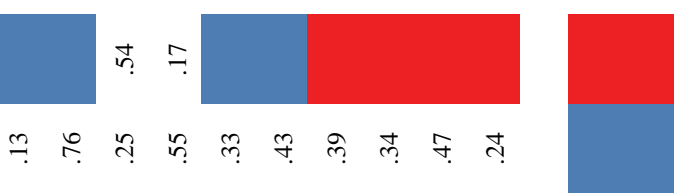

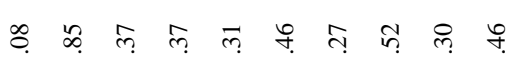

$\stackrel{\imath}{*}$ d
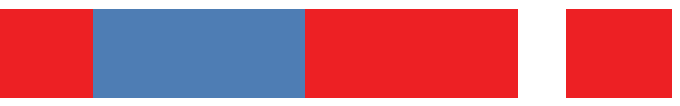

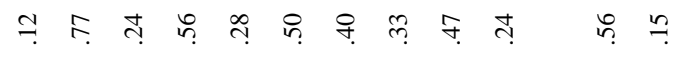

ศิ

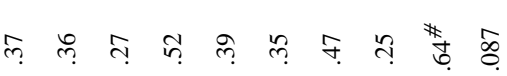

$\bigoplus$ ๘

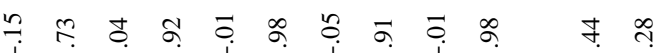

†े ๖ํํ

ๆ क्ष

ธ.

ำ

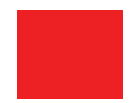

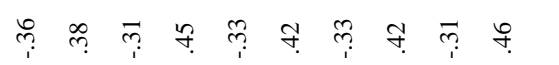

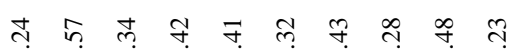

in 9

吝

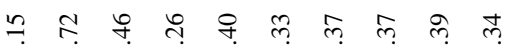

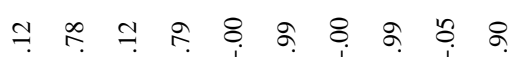
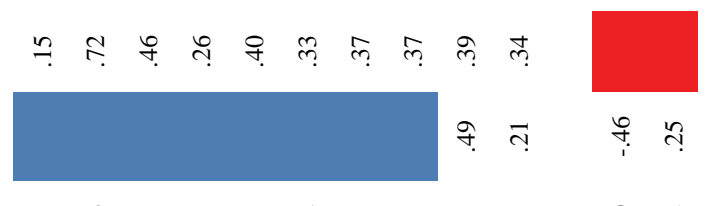

है $\stackrel{-}{4}$ ণิ

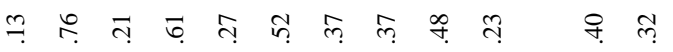

ชิ ำๆ

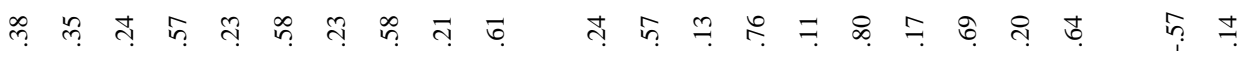

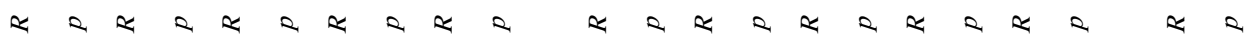

立

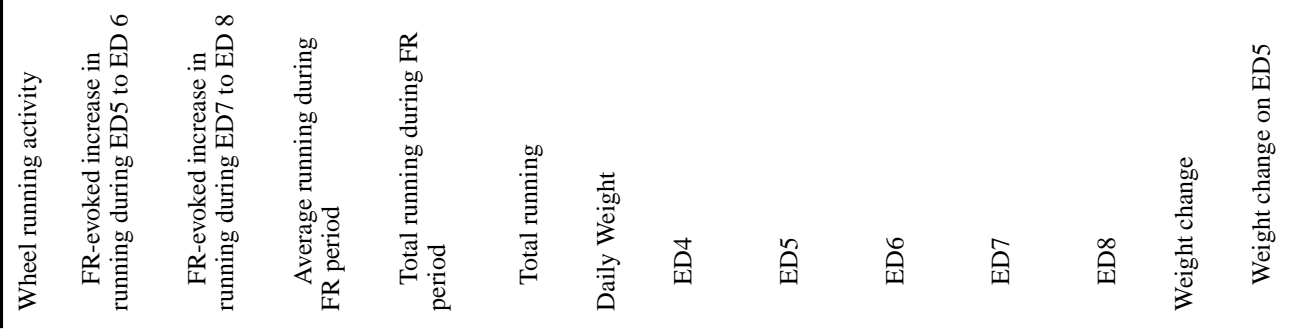

Brain Struct Funct. Author manuscript; available in PMC 2018 July 01. 


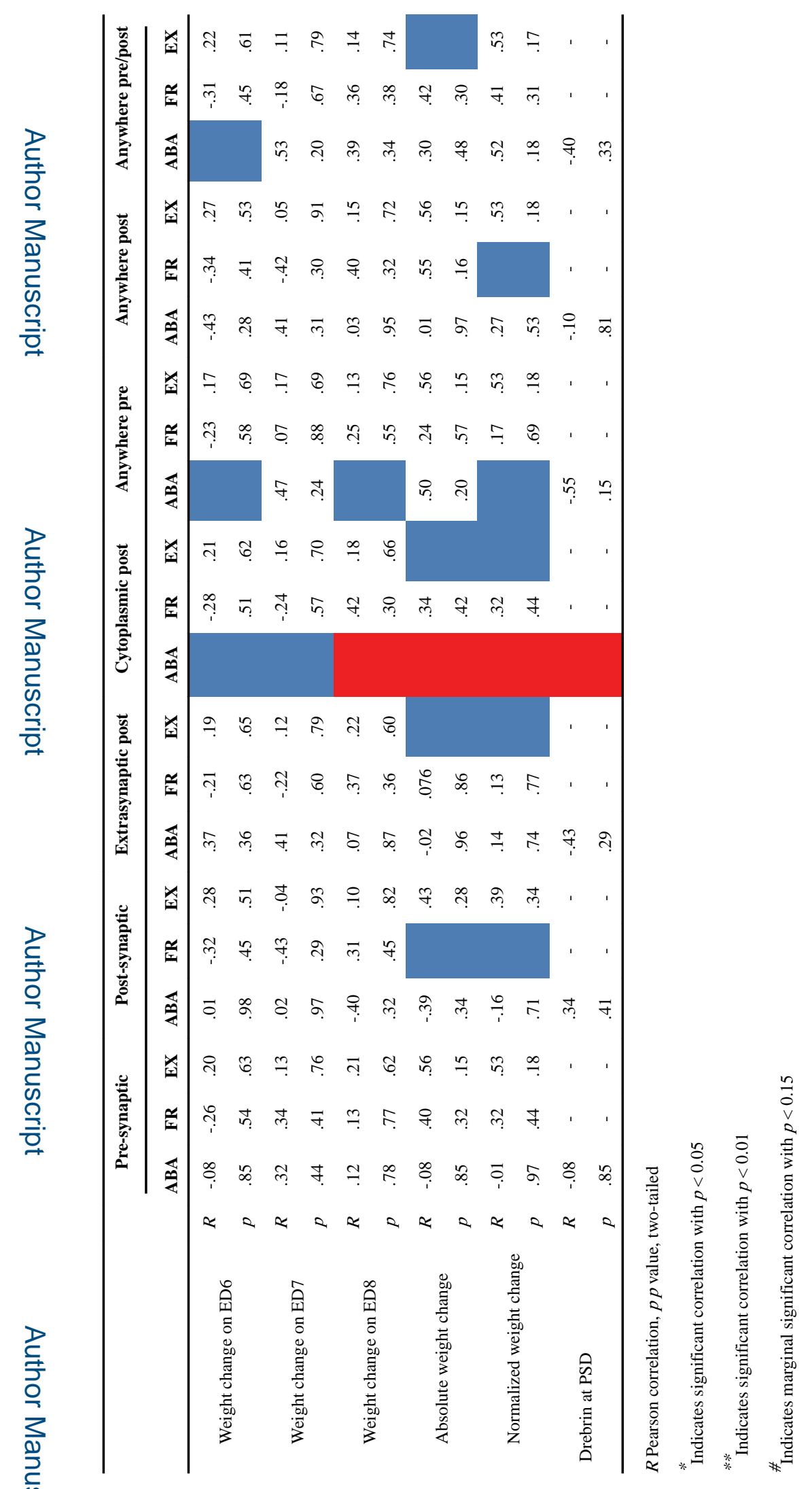

Brain Struct Funct. Author manuscript; available in PMC 2018 July 01. 


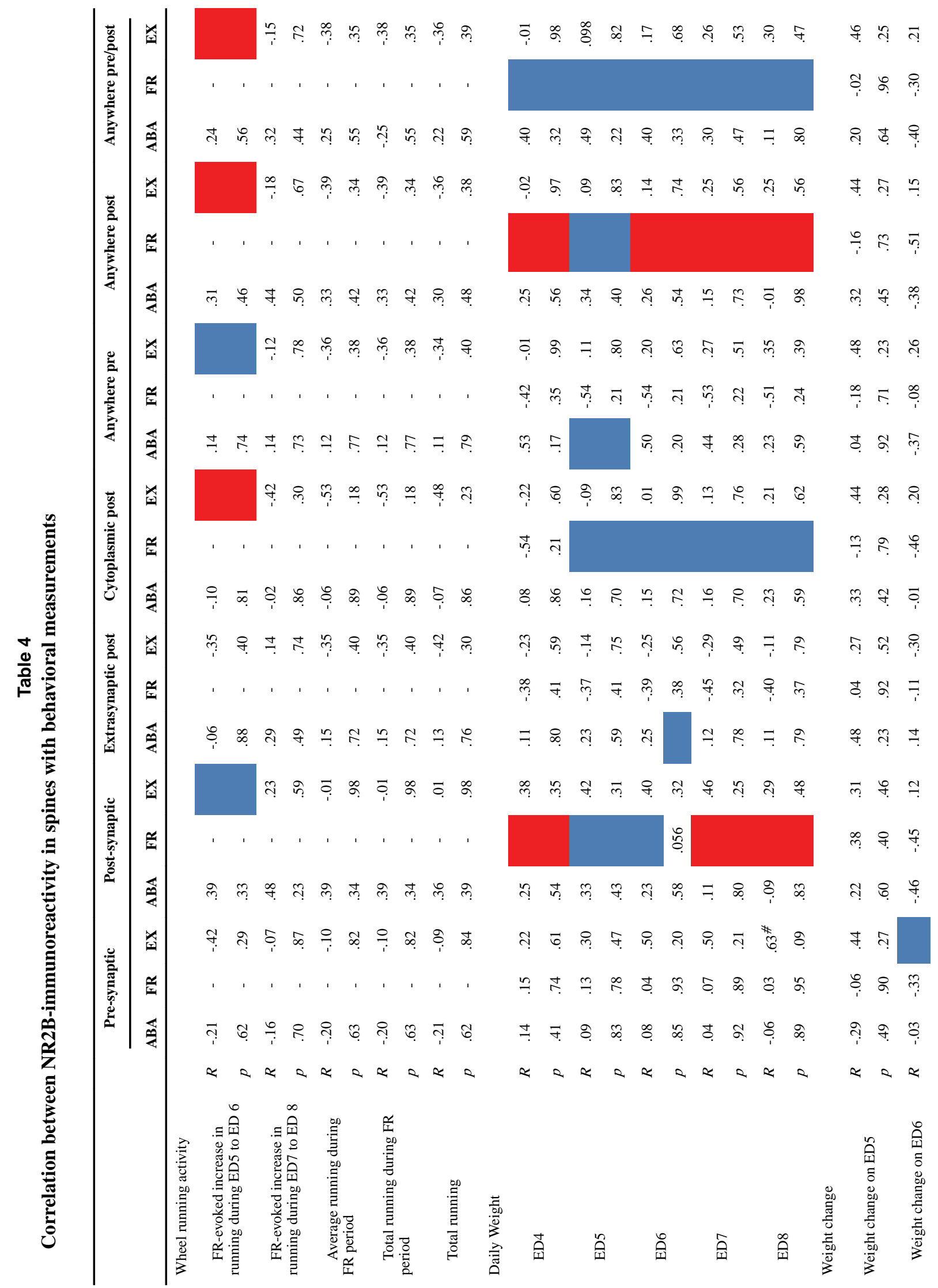

Brain Struct Funct. Author manuscript; available in PMC 2018 July 01. 


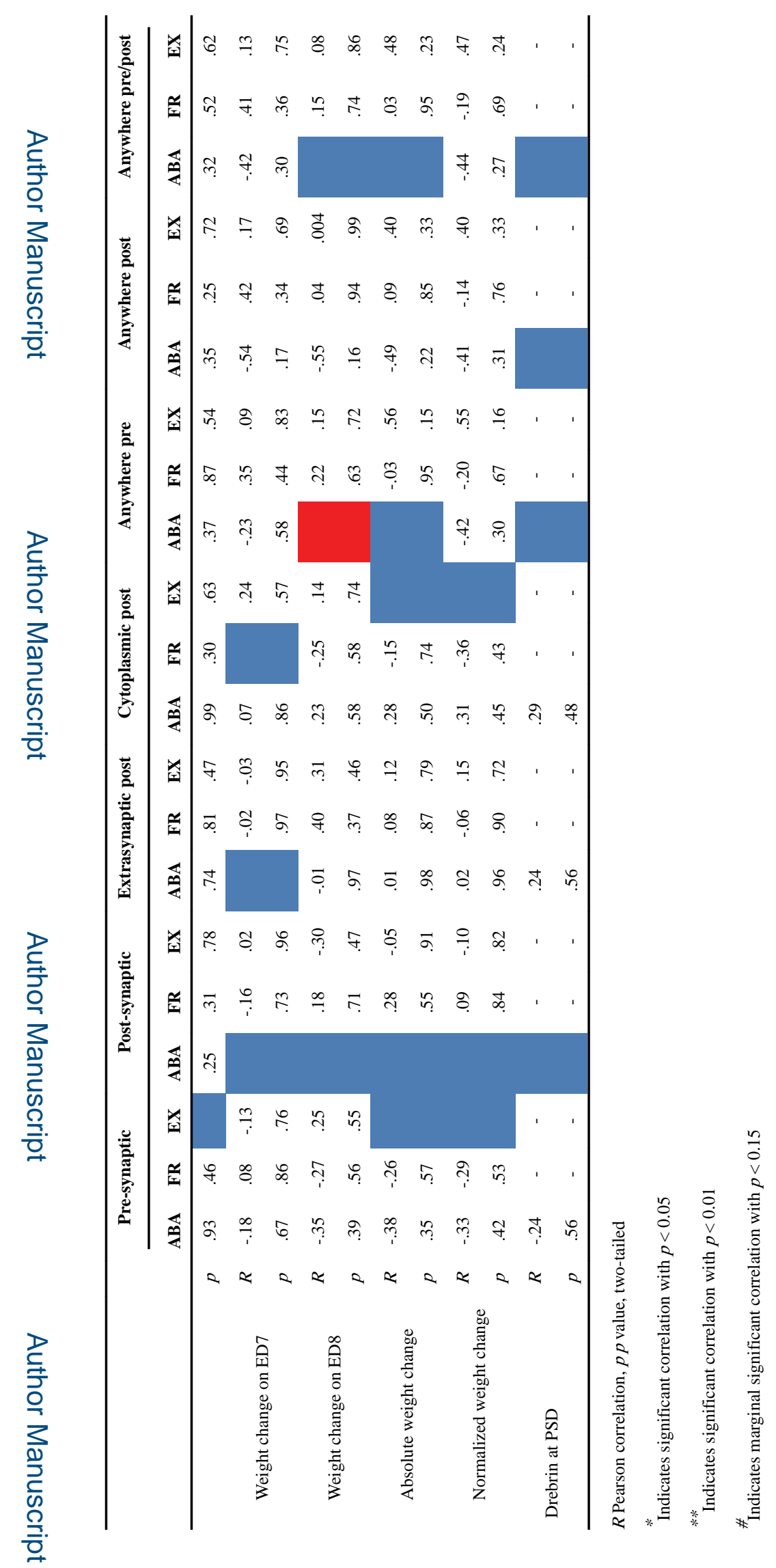

Brain Struct Funct. Author manuscript; available in PMC 2018 July 01. 